\title{
The Surface Brightness-color Relations Based on Eclipsing Binary Stars: Toward Precision Better than 1\% in Angular Diameter Predictions
}

\author{
Dariusz Graczyk $^{1,2,3}$, Piotr Konorski ${ }^{4}$, Grzegorz Pietrzyński $^{2,3}$, Wolfgang Gieren ${ }^{1,2}$, Jesper Storm ${ }^{5}$, Nicolas Nardetto ${ }^{6}$, \\ Alexandre Gallenne ${ }^{7}$, Pierre F. L. Maxted ${ }^{8}$, Pierre Kervella, ${ }^{9}, 10$, and Zbigniew Kołaczkowski ${ }^{11}$ \\ ${ }^{1}$ Millennium Institute of Astrophysics (MAS), Chile \\ ${ }^{2}$ Universidad de Concepción, Departamento de Astronomia, Casilla 160-C, Concepción, Chile; darek@astro-udec.cl \\ ${ }^{3}$ Centrum Astronomiczne im. Mikołaja Kopernika (CAMK), PAN, Bartycka 18, 00-716 Warsaw, Poland; darek@ncac.torun.pl \\ ${ }^{4}$ Obserwatorium Astronomiczne, Uniwersytet Warszawski, Al. Ujazdowskie 4, 00-478, Warsaw, Poland \\ ${ }_{5}^{5}$ Leibniz-Institut für Astrophysik Potsdam, An der Sternwarte 16, D-14482 Potsdam, Germany \\ ${ }^{6}$ Université Côte d'Azur, Observatoire de la Côte d'Azur, CNRS, Laboratoire Lagrange, UMR7293, Nice, France \\ ${ }^{7}$ European Southern Observatory, Alonso de Córdova 3107, Casilla 19001, Santiago 19, Chile \\ ${ }^{8}$ Astrophysics Group, Keele University, Staffordshire, ST5 5BG, UK \\ ${ }^{9}$ Unidad Mixta Internacional Franco-Chilena de Astronomía (CNRS UMI 3386), Departamento de Astronomía, Universidad de Chile, Camino El Observatorio 1515, \\ Las Condes, Santiago, Chile \\ ${ }^{10}$ LESIA (UMR 8109), Observatoire de Paris, PSL Research University, CNRS, UPMC, Université Paris-Diderot, 5 place Jules Janssen, F-92195 Meudon, France \\ ${ }^{11}$ Instytut Astronomiczny, Uniwersytet Wrocławski, Kopernika 11, 51-622 Wrocław, Poland \\ Received 2016 November 29; revised 2017 January 24; accepted 2017 January 25; published 2017 February 27
}

\begin{abstract}
In this study we investigate the calibration of surface brightness-color (SBC) relations based solely on eclipsing binary stars. We selected a sample of 35 detached eclipsing binaries with trigonometric parallaxes from Gaia DR1 or Hipparcos whose absolute dimensions are known with an accuracy better than $3 \%$ and that lie within $0.3 \mathrm{kpc}$ from the Sun. For the purpose of this study, we used mostly homogeneous optical and near-infrared photometry based on the Tycho-2 and 2MASS catalogs. We derived geometric angular diameters for all stars in our sample with a precision better than $10 \%$, and for 11 of them with a precision better than $2 \%$. The precision of individual angular diameters of the eclipsing binary components is currently limited by the precision of the geometric distances ( $\sim 5 \%$ on average). However, by using a subsample of systems with the best agreement between their geometric and photometric distances, we derived the precise SBC relations based only on eclipsing binary stars. These relations have precisions that are comparable to the best available SBC relations based on interferometric angular diameters, and they are fully consistent with them. With very precise Gaia parallaxes becoming available in the near future, angular diameters with a precision better than $1 \%$ will be abundant. At that point, the main uncertainty in the total error budget of the SBC relations will come from transformations between different photometric systems, disentangling of component magnitudes, and for hot OB stars, the main uncertainty will come from the interstellar extinction determination. We argue that all these issues can be overcome with modern high-quality data and conclude that a precision better than $1 \%$ is entirely feasible.
\end{abstract}

Key words: binaries: eclipsing - solar neighborhood - stars: fundamental parameters

\section{Introduction}

The surface brightness-color (SBC) relations play a fundamental role in predicting angular diameters of stars and serve as an almost perfect tool for deriving precise distances to eclipsing binary stars. They have also been extremely useful in BaadeWesselink techniques to determine the distances to classical Cepheid stars (e.g., Gieren et al. 1995; Fouqué \& Gieren 1997; Storm et al. 2011). The SBC relations are commonly calibrated based on direct stellar angular diameters measured by means of ground-based interferometry (e.g., Kervella et al. 2004; Di Benedetto 2005; Boyajian et al. 2014; Challouf et al. 2014). The precision of the SBC relations is gradually improving thanks to the ever-growing number of stars with interferometric angular diameters and to improvements in dealing with the limb darkening.

Eclipsing binaries with known trigonometric parallaxes can also be used to derive the SBC relation. This idea was first formulated and used by Lacy (1977): the combination of a geometric distance and stellar radius immediately provides an angular diameter of a component of an eclipsing binary. This can later be used to derive a dependence of the radiative flux scale on color, expressed in terms of the surface brightness parameter or the effective temperature. Deriving angular diameters of eclipsing binary stars is significantly more complex than determining an angular diameter of a single star with interferometry. However, using eclipsing binaries has mainly one important advantage: good control of the limbdarkening uncertainties, at least when the light curves are of sufficient quality (e.g., Popper 1984). Early attempts were constrained to the color $V-R$ (Lacy 1977; Barnes et al. 1978; Popper 1980) and were based on only three eclipsing binary systems with secure trigonometric parallaxes.

When Hipparcos parallaxes became available, Popper (1998) analyzed 14 well-detached eclipsing binaries with the most accurate parallaxes and absolute dimensions to compare radiative flux scales defined by interferometry and eclipsing binary systems. However, this analysis was made only for $(B-V)$ color and included eclipsing binaries with a significant amount of chromospheric activity. Nonetheless, Popper (1998) concluded that the SBC relation based on non-active eclipsing binaries seemed to be complementary to that based on interferometric angular diameters. Kruszewski \& Semeniuk (1999) developed the idea of using a large number of eclipsing binaries with geometric distances from Hipparcos to precisely 
Table 1

Basic Data of the Selected Detached Eclipsing Binaries

\begin{tabular}{|c|c|c|c|c|c|c|c|c|c|}
\hline Name & $\begin{array}{l}\text { Tycho-2 } \\
\text { ID }\end{array}$ & $\begin{array}{l}\text { R.A.2000 } \\
\text { h:m:s }\end{array}$ & $\begin{array}{c}\text { Decl. }_{2000} \\
\text { deg:m:s }\end{array}$ & $\begin{array}{c}V^{\mathrm{a}} \\
\mathrm{mag}\end{array}$ & $\begin{array}{l}\text { Spectral } \\
\text { Type }\end{array}$ & $\begin{array}{l}\text { References } \\
\text { SpT }\end{array}$ & $\begin{array}{c}\text { Orbital } \\
\text { Period (d) }\end{array}$ & $\begin{array}{c}\text { References } \\
\text { OrP }\end{array}$ & $\begin{array}{c}\text { Parallax } \\
\text { mas }\end{array}$ \\
\hline YZ Cas & $4307-2167-1$ & $00: 45: 39.077$ & $+74: 59: 17.06$ & $5.653 \pm 0.015$ & $\mathrm{~A} 2 \mathrm{~m}+\mathrm{F} 2 \mathrm{~V}$ & 1 & 4.4672235 & 36 & $10.30 \pm 0.49$ \\
\hline AI Phe & $8032-0625-1$ & 01:09:34.195 & $-46: 15: 56.09$ & $8.610 \pm 0.019$ & $\mathrm{~F} 8 \mathrm{~V}+\mathrm{K} 0 \mathrm{IV}$ & 8 & 24.592483 & 40 & $5.94 \pm 0.24$ \\
\hline V505 Per & $3690-0536-1$ & 02:21:12.964 & $+54: 30: 36.28$ & $6.889 \pm 0.016$ & $\mathrm{~F} 5 \mathrm{~V}+\mathrm{F} 5 \mathrm{~V}$ & 2 & 4.222020 & 2 & $15.56 \pm 0.32$ \\
\hline AL Ari & $0645-1107-1$ & $02: 42: 36.341$ & $+12: 44: 07.77$ & $9.223 \pm 0.034$ & $\mathrm{~F} 5 \mathrm{~V}+\mathrm{G} 4 \mathrm{~V}$ & 3 & 3.7474543 & 3 & $7.11 \pm 0.37$ \\
\hline V570 Per & $3314-1225-1$ & 03:09:34.944 & $+48: 37: 28.69$ & $8.091 \pm 0.018$ & $\mathrm{~F} 3 \mathrm{~V}+\mathrm{F} 5 \mathrm{~V}$ & 4 & 1.9009382 & 4 & $7.85 \pm 0.26$ \\
\hline TZ For & 7026-0633-1 & 03:14:40.093 & $-35: 33: 27.60$ & $6.888 \pm 0.016$ & F7IV+G8III & 5 & 75.66647 & 37 & $5.379 \pm 0.055^{\mathrm{b}}$ \\
\hline V1229 $\mathrm{Tau}^{\mathrm{c}}$ & $1800-1622-1$ & 03:47:29.454 & $+24: 17: 18.04$ & $6.807 \pm 0.017$ & $\mathrm{~A} 0 \mathrm{~V}+\mathrm{Am}$ & 6 & 2.46113408 & 38 & $7.57 \pm 0.40$ \\
\hline V1094 Tau & $1263-0642-1$ & 04:12:03.593 & $+21: 56: 50.55$ & $8.981 \pm 0.031$ & $\mathrm{G} 0 \mathrm{~V}+\mathrm{G} 2 \mathrm{~V}$ & 16 & 8.9885474 & 45 & $8.26 \pm 0.25$ \\
\hline CD Tau & $1291-0292-1$ & $05: 17: 31.153$ & $+20: 07: 54.63$ & $6.768 \pm 0.016$ & F6V+F6V & 7 & 3.435137 & 39 & $13.56 \pm 0.38$ \\
\hline EW Ori & 0104-1206-1 & 05:20:09.147 & $+02: 02: 39.97$ & $9.902 \pm 0.043^{\mathrm{d}}$ & $\mathrm{F} 8 \mathrm{~V}+\mathrm{G} 0 \mathrm{~V}$ & 16,47 & 6.9368432 & 47 & $5.48 \pm 0.23$ \\
\hline UX Men & 9378-0190-1 & 05:30:03.184 & $-76: 14: 55.35$ & $8.251 \pm 0.017$ & $\mathrm{~F} 8 \mathrm{~V}+\mathrm{F} 8 \mathrm{~V}$ & 9,5 & 4.181100 & 41 & $9.72 \pm 0.21$ \\
\hline TZ Men & 9496-0590-1 & 05:30:13.886 & $-84: 47: 06.37$ & $6.186 \pm 0.016$ & $\mathrm{~A} 0 \mathrm{~V}+\mathrm{A} 8 \mathrm{~V}$ & 10,5 & 8.56900 & 10 & $8.02 \pm 0.49$ \\
\hline$\beta$ Aur & $2924-2742-1$ & $05: 59: 31.723$ & $+44: 56: 50.76$ & $1.900 \pm 0.020^{\mathrm{e}}$ & $\mathrm{A} 1 \mathrm{mIV}+\mathrm{A} 1 \mathrm{mIV}$ & 11,57 & 3.960047 & 42 & $40.21 \pm 0.23^{\mathrm{f}}$ \\
\hline RR Lyn & $3772-2770-1$ & $06: 26: 25.836$ & $+56: 17: 06.35$ & $5.558 \pm 0.015$ & $\mathrm{~A} 6 \mathrm{mIV}+\mathrm{F} 0 \mathrm{~V}$ & 12 & 9.945074 & 12 & $13.34 \pm 0.60^{\mathrm{f}}$ \\
\hline WW Aur & $2426-0345-1$ & $06: 32: 27.185$ & $+32: 27: 17.63$ & $5.832 \pm 0.016$ & $\mathrm{~A} 4 \mathrm{~m}+\mathrm{A} 5 \mathrm{~m}$ & 13 & 2.5250194 & 43 & $11.03 \pm 0.50$ \\
\hline HD 71636 & 2489-1972-1 & 08:29:56.311 & $+37: 04: 15.48$ & $7.903 \pm 0.018$ & $\mathrm{~F} 2 \mathrm{~V}+\mathrm{F} 5 \mathrm{~V}$ & 14 & 5.013292 & 14 & $8.40 \pm 0.40$ \\
\hline VZ Hya & 4874-0811-1 & 08:31:41.413 & $-06: 19: 07.56$ & $8.953 \pm 0.027^{\mathrm{g}}$ & $\mathrm{F} 3 \mathrm{~V}+\mathrm{F} 5 \mathrm{~V}$ & 21 & 2.9043002 & 51 & $6.94 \pm 0.24$ \\
\hline $\mathrm{KX}$ Cnc & 2484-0592-1 & $08: 42: 46.211$ & $+31: 51: 45.37$ & $7.192 \pm 0.017$ & F9V+F9V & 15 & 31.2197874 & 44 & $20.54 \pm 0.38$ \\
\hline PT Vel & $7690-2859-1$ & 09:10:57.720 & $-43: 16: 02.93$ & $7.027 \pm 0.016$ & $\mathrm{~A} 0 \mathrm{~V}+\mathrm{F} 0$ & 17 & 1.802008 & 17 & $6.15 \pm 0.45$ \\
\hline KW Hya & 4891-1371-1 & 09:12:26.044 & $-07: 06: 35.38$ & $6.100 \pm 0.016$ & $\mathrm{~A} 5 \mathrm{~m}+\mathrm{F} 0 \mathrm{~V}$ & 18 & 7.750469 & 46 & $11.53 \pm 0.42^{\mathrm{f}}$ \\
\hline RZ Cha & 9422-0104-1 & $10: 42: 24.104$ & $-82: 02: 14.19$ & $8.091 \pm 0.018$ & $\mathrm{~F} 5 \mathrm{~V}+\mathrm{F} 5 \mathrm{~V}$ & 20 & 2.832084 & 48 & $5.68 \pm 0.26$ \\
\hline FM Leo & 0263-0727-1 & $11: 12: 45.095$ & $+00: 20: 52.83$ & $8.460 \pm 0.021$ & F7V+F7V & 21 & 6.728606 & 49 & $7.00 \pm 0.32$ \\
\hline GG Lup & 7826-3079-1 & $15: 18: 56.376$ & $-40: 47: 17.60$ & $5.603 \pm 0.015$ & $\mathrm{~B} 7 \mathrm{~V}+\mathrm{B} 9 \mathrm{~V}$ & 23 & 1.8495927 & 50 & $5.96 \pm 0.30^{\mathrm{f}}$ \\
\hline V335 Ser & 0353-0301-1 & $15: 59: 05.756$ & $+00: 35: 44.55$ & $7.490 \pm 0.017$ & $\mathrm{~A} 1 \mathrm{~V}+\mathrm{A} 3 \mathrm{~V}$ & 19 & 3.4498837 & 19 & $4.74 \pm 0.30$ \\
\hline WZ Oph & 0977-0216-1 & 17:06:39.042 & $+07: 46: 57.78$ & $9.126 \pm 0.024$ & $\mathrm{~F} 8 \mathrm{~V}+\mathrm{F} 8 \mathrm{~V}$ & 24,25 & 4.183507 & 51 & $6.61 \pm 0.24$ \\
\hline FL Lyr & $3542-1492-1$ & $19: 12: 04.862$ & $+46: 19: 26.86$ & $9.366 \pm 0.026$ & $\mathrm{~F} 8 \mathrm{~V}+\mathrm{G} 8 \mathrm{~V}$ & 27 & 2.1781542 & 27 & $7.25 \pm 0.22$ \\
\hline UZ Dra & 4444-1595-1 & $19: 25: 55.045$ & $+68: 56: 07.15$ & $9.601 \pm 0.028$ & $\mathrm{~F} 7 \mathrm{~V}+\mathrm{G} 0 \mathrm{~V}$ & 22 & 3.261302 & 52 & $5.21 \pm 0.25$ \\
\hline
\end{tabular}




\begin{tabular}{|c|c|c|c|c|c|c|c|c|c|}
\hline Name & $\begin{array}{c}\text { Tycho-2 } \\
\text { ID }\end{array}$ & $\begin{array}{l}\text { R.A.2000 } \\
\text { h:m:s }\end{array}$ & $\begin{array}{c}\text { Decl. }_{2000} \\
\text { deg:m:s }\end{array}$ & $\begin{array}{c}V^{\mathrm{a}} \\
\mathrm{mag}\end{array}$ & $\begin{array}{l}\text { Spectral } \\
\text { Type }\end{array}$ & $\begin{array}{l}\text { References } \\
\text { SpT }\end{array}$ & $\begin{array}{l}\text { Orbital } \\
\text { Period (d) }\end{array}$ & $\begin{array}{l}\text { References } \\
\text { OrP }\end{array}$ & $\begin{array}{l}\text { Parallax } \\
\text { mas }\end{array}$ \\
\hline V4089 Sgr & 7936-2270-1 & 19:34:08.486 & $-40: 02: 04.70$ & $5.907 \pm 0.016$ & $\mathrm{~A} 2 \mathrm{IV}+\mathrm{A} 7 \mathrm{~V}$ & 28,29 & 4.6270956 & 29 & $6.75 \pm 0.49$ \\
\hline V1143 Cyg & 3938-1983-1 & 19:38:41.184 & $+54: 58: 25.65$ & $5.889 \pm 0.015$ & $\mathrm{~F} 5 \mathrm{~V}+\mathrm{F} 5 \mathrm{~V}$ & 30,31 & 7.640742 & 54 & $24.75 \pm 0.35$ \\
\hline MY Cyg & $2680-1529-1$ & 20:20:03.390 & $+33: 56: 35.02$ & $8.341 \pm 0.019$ & $\mathrm{~F} 0 \mathrm{~m}+\mathrm{F} 0 \mathrm{~m}$ & 32 & 4.0051870 & 55 & $3.95 \pm 0.24$ \\
\hline EI Cep & 4599-0082-1 & 21:28:28.206 & $+76: 24: 12.59$ & $7.600 \pm 0.017$ & $\mathrm{~F} 3 \mathrm{~V}+\mathrm{F} 1 \mathrm{~V}$ & 33 & 8.4393522 & 33 & $5.07 \pm 0.24$ \\
\hline VZ Cep & $4470-1334-1$ & 21:50:11.135 & $+71: 26: 38.30$ & $9.717 \pm 0.009^{\mathrm{h}}$ & $\mathrm{F} 3 \mathrm{~V}+\mathrm{G} 4 \mathrm{~V}$ & 26 & 1.1833638 & 26 & $3.88 \pm 0.35$ \\
\hline LL Aqr & 5236-0883-1 & 22:34:42.152 & $-03: 35: 58.17$ & $9.243 \pm 0.037^{\mathrm{i}}$ & $\mathrm{F} 8 \mathrm{~V}+\mathrm{G} 2 \mathrm{~V}$ & 34 & 20.178321 & 56 & $7.75 \pm 0.27$ \\
\hline EF Aqr & 5248-1030-1 & 23:01:19.088 & $-06: 26: 15.35$ & $9.885 \pm 0.022^{\mathrm{j}}$ & $\mathrm{F} 8 \mathrm{~V}+\mathrm{G} 8 \mathrm{~V}$ & 15 & 2.8535721 & 53 & $5.06 \pm 0.50$ \\
\hline V821 Cas & 4001-1445-1 & 23:58:49.175 & $+53: 40: 19.81$ & $8.286 \pm 0.017$ & $\mathrm{~A} 1 \mathrm{~V}+\mathrm{A} 4$ & 35 & 1.7697397 & 35 & $3.61 \pm 0.30$ \\
\hline
\end{tabular}

Notes.

${ }^{a}$ Tycho-2 $V_{T}$ magnitudes from $\mathrm{H} ø \mathrm{~g}$ et al. (2000) converted into Johnson $V$ magnitudes using the transformation given by Bessell (2000).

${ }^{\mathrm{b}}$ Orbital parallax from Gallenne et al. (2016), Hipparcos value $\varpi=5.75 \pm 0.51$ mas, Gaia value $\varpi=5.44 \pm 0.25$ mas.

${ }^{\mathrm{c}}$ HD 23642, in the Pleiades cluster.

${ }^{\mathrm{d}}$ Clausen et al. (2010).

e Mermilliod (1991).

${ }^{\mathrm{f}}$ Hipparcos parallax (van Leeuwen 2007).

${ }^{\mathrm{g}}$ Clausen et al. (2008a).

${ }^{\mathrm{h}}$ Lacy (2002).

${ }^{\mathrm{i}}$ Graczyk et al. (2016).

${ }^{\mathrm{j}}$ Vos et al. (2012).

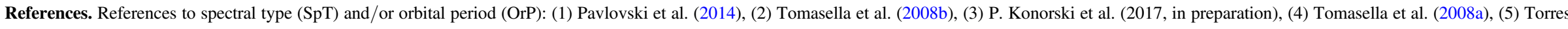

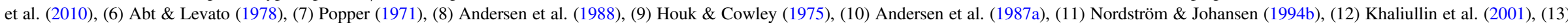

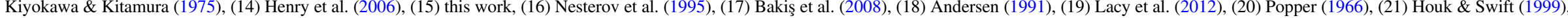

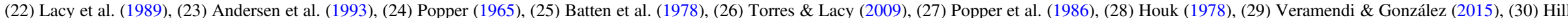

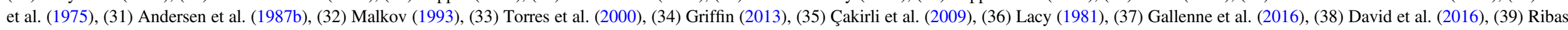

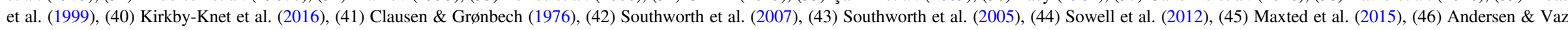

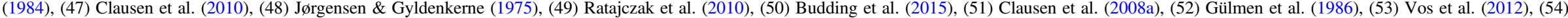

Giménez \& Margrave (1985), (55) Tucker et al. (2009), (56) Southworth (2013), (57) Lyubimkov et al. (1996). 
calibrate the SBC relations. They compiled an extensive list of promising eclipsing binaries in the solar neighborhood (up to $200 \mathrm{pc}$ ). Soon after, Semeniuk (2001) derived the SBC relation from a sample of 13 eclipsing binary stars with Hipparcos parallaxes and Strömgren photometry. The calibration was made for the $(b-y)$ color and compared with the relation by Popper (1998), which was mostly based on interferometric and lunar occultation angular diameter measurements. The samples agreed well, but the derived SBC relation had very large scatter.

The usefulness of eclipsing binaries for distance measurements was investigated by Jerzykiewicz (2001) by comparison of corrected trigonometric parallaxes and photometric distances, with the conclusion that EBs are excellent standard candles. Smalley et al. (2002) used 15 eclipsing binary stars with Hipparcos parallaxes to derive a fundamental temperature scale for A-type stars, and Bilir et al. (2008) presented a brief analysis of using eclipsing binary stars to calibrate the absolute magnitudes of stars as a function of some intrinsic colors. The most recent application of eclipsing binaries to derive the SBC relations known to us is the work by Bonneau et al. (2006), where the SBC relation was calibrated against $(V-K)$ color, but these authors used photometric distances to derive angular diameters (see our Section 3.6). Recently, Stassun \& Torres (2016a, 2016b) used more than 100 eclipsing binaries to investigate possible systematics in recent Gaia DR1 parallaxes (Gaia Collaboration et al. 2016) and concluded that a likely systematic shift of -0.25 mas is presented in Gaia parallaxes. This shift is consistent with the systematic global error of 0.3 mas in the DR1 that was announced by the Gaia team.

The list of eclipsing binary systems reported by Kruszewski \& Semeniuk (1999) is the basis for our program of investigating eclipsing binaries and deriving the SBC relations. The first paper from our program was devoted to the IO Aqr system (Graczyk et al. 2015) and showed that unrecognized triples may bias the derivation of the SBC relations. Although the maximum-light contribution of the third component of IO Aqr is low and relatively well determined, the SBC calibrations have substantial problems to reach a precision of about $1 \%$ for this system. In a following paper (Gallenne et al. 2016) we derived a very precise orbital parallax to TZ For that allowed us to perform a preliminary check of the precision of existing SBC relations. Our parallax measurement to $\mathrm{TZ}$ For is in perfect agreement with the photometric distance and the Gaia DR1 parallax. The work on TZ For is part of our larger effort to determine very precise dynamical parallaxes to a number of long-period eclipsing binaries.

Here we present in detail the method of deriving the SBC relations based on eclipsing binary stars, and for the first time, we publish precise relations that are based solely on eclipsing binaries. Section 2 characterizes a sample of systems and describes the selection criteria and data we used. In Section 3 we present the method outline of our analysis. Section 4 contains results, and these are discussed in Section 5. The last section is devoted to final remarks.

\section{The Sample}

For the purpose of our work, we use a volume-limited $(d<300 \mathrm{pc})$ sample of detached eclipsing binaries with published high-quality light curve and radial velocity solutions. The sample is assumed to contain standard eclipsing binary systems for the purpose of accurate distance determination or validation and surface brightness calibration. We made an extensive search for suitable systems in the literature using the SIMBAD database (Wenger et al. 2000) and NASA ADS. Useful guidance in this context is provided by the compilations published by Kruszewski \& Semeniuk (1999), Bilir et al. (2008), and Torres et al. (2010) and more recently by Eker et al. (2014), Southworth (2015), and Stassun \& Torres (2016a). The final sample contains 34 systems and additionally AL Ari, a system for which our new analysis is as yet unpublished (P. Konorski et al. 2017, in preparation). Our intention is for the sample to serve as a reference catalog for very precise determinations of photometric distances, angular diameters, and the surface brightness. We place very strict conditions on including an eclipsing binary in our sample. As a part of the selection procedure, we extensively checked the consistency of published physical parameters for every candidate system and in some cases recalculated fundamental parameters to make them more concordant with the observables. Table 1 presents the basic information about selected eclipsing binaries. The criteria are described below in detail.

\subsection{Proximity Effects}

No proximity effects larger than 0.03 mag. Although semidetached or even contact-configuration eclipsing binaries were suggested as good distance indicators (e.g., Wyithe \& Wilson 2002; Wilson et al. 2010), our experience shows that their physical parameters are usually much more model dependent and thus less reliable than those coming from an analysis of detached eclipsing binaries. In fact, only welldetached systems offer a very simple geometry where both stars can be treated as almost perfect spheres. This simplifies the analysis, as magnitudes and colors of the system are virtually constant outside eclipses.

\subsection{Intrinsic Variability}

No intrinsic variability amplitude larger than $0.04 \mathrm{mag}$. Larger variability (spots, pulsations) over a given threshold may lead to some bias in the estimation of true photometric indices on a level of $>2 \%$, therefore we removed all systems with an active or pulsating component from our sample (e.g., RS CVn stars, chromospheric activity). The only system we retained is EF Aqr, which shows some spot activity on a secondary, but only small changes in the combined out-ofeclipse light (Vos et al. 2012).

\subsection{Absolute Dimensions}

Precision better than 3\%. For the purpose of surface brightness calibration, a knowledge of the physical radii is fundamental because when it is combined with a distance, it gives the angular diameters. We chose known systems with the most precise absolute dimensions. The average precision of the radii determination in the sample is $\sigma_{R} / R=1.2 \%$. With this sample, the currently avaliable Gaia parallaxes can be used. For future Gaia releases, which are expected to have a precision better than $1 \%$ for all stars in our sample (de Bruijne et al. 2014), some of the systems will need to be reanalyzed in order to achieve a precision better than $2 \%$ of the radii determination or may even have to be removed from the sample. These systems are V1229 Tau, FM Leo, FL Lyr, MY Cyg, VZ Cep, and V821 Cas. 


\subsection{Geometric Distance}

Precision better than $10 \%$ within 300 pc horizon. We used trigonometric parallaxes from the recent Gaia Data Release 1 (Gaia Collaboration et al. 2016), augmented with Hipparcos parallaxes (van Leeuwen 2007) for some bright and nearby systems. There are still only a few eclipsing binaries in our sample with high-precision trigonometric parallaxes (fractional uncertainty $\sigma_{\pi} / \pi<2 \%$ ). In the case of one system, TZ For, we used the orbital parallax determined by Gallenne et al. (2016), which is more precise by a factor of 5 than the Gaia DR1 parallax.

\subsection{Temperature}

Effective temperatures known to within 5\%. We use them to build precise models of the systems and to calculate infrared light ratios. In this work we also used temperatures to derive photometric distances (by the flux scaling) as proxies of the true geometric distances. In general, temperatures are important for determining auxiliary parameters (e.g., limb darkening) during light-curve analysis, and we therefore preferred systems with well-determined radiative properties.

\subsection{Multiplicity}

We excluded systems with confirmed third light in photometry or spectroscopy, or systems in which known close bright companions affect the photometric indexes. CD Tau has a close K-type companion at a distance of $\sim 10^{\prime \prime}$. The light of the companion is present in the optical light curves analyzed by Ribas et al. (1999), but it was carefully accounted for in their analysis. The companion is far enough away to not influence the Tycho or 2MASS magnitudes. The system AI Phe also has a fainter visual companion $\left(11^{\prime \prime}\right)$, the presence of which was accounted for by Kirkby-Knet et al. (2016) in their analysis. The case of AI Phe is more complex because this system has another even closer invisible companion that induces acceleration on the main binary system (M. Konacki 2017, private communication). The nature of this companion is currently uncertain, but spectroscopic data suggest that it is an M-type dwarf. In this case, its luminosity can be completely neglected (even in the NIR), and we included this system in our sample. RR Lyn is a proposed triple system with a companion of 0.1 $M_{\odot} \quad$ (Khaliullin \& Khaliullina 2002). Even though the companion may be confirmed with future spectroscopic monitoring, no third light is currently visible in high-quality light curves (e.g., Khaliullin et al. 2001), and we retained this system in our sample.

\subsection{Photometry}

We decided to use homogenous non-saturated optical/ infrared photometry from Tycho-2 and the Two Micron All Sky Survey.

\subsubsection{Optical}

We downloaded the optical $B V$ Tycho-2 photometry (Høg et al. 2000) of the eclipsing binaries from Vizier (Ochsenbein et al. 2000). ${ }^{12}$ The star that is by far the brightest in our sample is $\beta$ Aur. It is the only star that lacks Tycho photometry. In this case, we used Johnson photometry from a compilation by

\footnotetext{
12 http://vizier.u-strasbg.fr:I/259/tyc2
}

Mermilliod (1991). Tycho photometry was subsequently transformed into the Johnson system using the method outlined by Bessell (2000). For six systems Tycho-2 photometry leads to unexplainable shifts in the temperatures and surface brightness parameter derived, and we therefore replaced it by more precise out-of-eclipse optical photometry from the literature.

\subsubsection{Near-infrared}

We downloaded NIR $J H K_{S}$ photometry of the Two Micron All Sky Survey (2MASS) (Skrutskie et al. 2006) from Vizier. ${ }^{13}$ Magnitudes were converted into the Johnson system using equations given in Bessell \& Brett (1988) and Carpenter (2001). ${ }^{14}$ The transformation equations are as follows:

$$
\begin{aligned}
K_{J}-K_{2 \mathrm{M}} & =0.037-0.017(J-K)_{2 \mathrm{M}}-0.007(V-K)_{2 \mathrm{M}} \\
(J-K)_{J} & =1.064(J-K)_{2 \mathrm{M}}+0.006 \\
(H-K)_{J} & =1.096(H-K)_{2 \mathrm{M}}-0.027
\end{aligned}
$$

2MASS photometry of $\beta$ Aur is saturated, and we used Johnson $J K$ photometry from a compilation by Ducati (2002). A lack of good NIR photometry forced us to remove the otherwise well-suited system $\psi$ Cen from the sample.

\section{Method}

\subsection{The Wilson-Devinney Model of the Systems}

For the purpose of obtaining homogenous parameters for the eclipsing binary sample, we decided to create a model of each system. The models were built using the Wilson-Devinney code version 2007 (Wilson \& Devinney 1971; Wilson 1979, 1990; van Hamme \& Wilson 2007), while parameters of the models were based on solutions published in the literature. None of the eclipsing binaries in our sample has infrared $J, H, K$ light curves that would be suitable for deriving direct light ratios in those bands. In order to calculate intrinsic infrared colors of the components of each system, we therefore employed eclipsing binary models based on optical light curves, and we extrapolated them into the infrared. This approach may introduce some bias; we discuss this later in this paper. All models were checked for internal consistency of the parameters, and we found that in many cases they had to be fine-tuned. In particular, the temperature ratio and the absolute temperature scale, which are crucial for precise prediction of infrared light ratios, were inspected carefully.

The procedure was as follows. For each system we collected orbital and photometric parameters from the most recent publications. The input parameters were the radial velocity semiamplitudes $K_{1,2}$, the orbital period $P$, three parameters describing the position of the orbit (the orbital inclination $i$, the eccentricity $e$, and the longitude of periastron $\omega$ ), the photometric relative radii $r_{1,2}$, and the effective temperatures $T_{1,2}$. These parameters were transformed into the semimajor axis of the system $a$, the mass ratio $q$, and into dimensionless Roche potentials $\Omega_{1,2}$ using the equations given in Torres et al. (2010) and Wilson (1979), i.e., parameters directly fitted or used within the WD program. We usually fixed the temperature of the primary star $T_{1}$ and then, using published light ratios in different photometric bands, we adjusted the temperature of the companion $T_{2}$. In some cases, however, we also rederived $T_{1}$ as

\footnotetext{
13 http://vizier.u-strasbg.fr:II/281/2mass6x

14 http://www.astro.caltech.edu/ jmc/2mass/v3/transformations /
} 
Table 2

Parameters of the Wilson-Devinney Models

\begin{tabular}{|c|c|c|c|c|c|c|c|c|c|c|c|c|c|c|}
\hline \multirow{3}{*}{$\begin{array}{l}\text { Eclipsing } \\
\text { Binary }\end{array}$} & \multicolumn{9}{|c|}{ Input Parameters } & \multirow{3}{*}{ Reference } & \multicolumn{4}{|c|}{ Model Parameters } \\
\hline & \multicolumn{2}{|c|}{ RV Semiamplitude } & \multicolumn{3}{|c|}{ Orientation of the Orbit } & \multicolumn{2}{|c|}{ Fractional Radius } & \multicolumn{2}{|c|}{ Effective Temperature } & & \multirow{2}{*}{$\begin{array}{c}\text { Semimajor } \\
\text { Axis }\left(R_{\odot}\right)\end{array}$} & \multirow{2}{*}{$\begin{array}{l}\text { Mass } \\
\text { Ratio }\end{array}$} & \multirow{2}{*}{$\Omega_{1}$} & \multirow{2}{*}{$\Omega_{2}$} \\
\hline & $K_{1}\left(\mathrm{~km} \mathrm{~s}^{-1}\right)$ & $K_{2}\left(\mathrm{~km} \mathrm{~s}^{-1}\right)$ & $e$ & $\omega(\mathrm{rad})$ & $i(\mathrm{deg})$ & $r_{1}$ & $r_{2}$ & $T_{1}(\mathrm{~K})$ & $T_{2}(\mathrm{~K})$ & & & & & \\
\hline YZ Cas & $73.05(19)$ & $124.78(27)$ & 0.0 & 0.0 & $88.33(7)$ & $0.14456(56)$ & $0.07622(33)$ & $9520(120)$ & $6880(240)$ & 2 & 17.4764 & 0.5854 & 7.5141 & 8.8912 \\
\hline AI Phe ${ }^{a}$ & $51.128(28)$ & $49.120(19)$ & $0.187(3)$ & $1.933(6)$ & $88.55(5)$ & $0.03845(35)$ & $0.06070(27)$ & $6175(150)$ & $5140(120)$ & $3,4,5,1$ & 47.8850 & 1.0409 & 27.290 & 18.361 \\
\hline V505 Per & $89.01(8)$ & $90.28(9)$ & 0.0 & 0.0 & $87.95(4)$ & $0.0860(9)$ & $0.0846(9)$ & 6512(21) & $6460(30)$ & 6 & 14.9724 & 0.9859 & 12.618 & 12.665 \\
\hline AL Ari & $76.98(13)$ & $98.38(21)$ & $0.051(3)$ & $1.20(2)$ & $89.48(6)$ & $0.1060(4)$ & $0.0696(3)$ & $6300(80)$ & 5412(80) & 7 & 12.9738 & 0.7825 & 10.269 & 12.411 \\
\hline V570 Per $^{\mathrm{b}}$ & $114.09(27)$ & $122.48(28)$ & 0.0 & 0.0 & $77.4(3)$ & $0.1675(31)$ & $0.1526(19)$ & $6842(30)$ & $6562(30)$ & 8,1 & 9.10738 & 0.9315 & 6.9200 & 7.1538 \\
\hline TZ For ${ }^{\mathrm{c}}$ & $40.868(11)$ & $38.900(22)$ & 0.0 & 0.0 & $85.66(4)$ & $0.03320(70)$ & $0.06972(92)$ & $6350(70)$ & 4930(30) & 9,10 & 119.650 & 1.0506 & 31.416 & 16.046 \\
\hline V1229 Tau & $99.02(27)$ & $140.86(36)$ & 0.0 & 0.0 & $78.2(1)$ & $0.1450(23)$ & $0.1262(37)$ & $9950(300)$ & $7640(300)$ & 11,12 & 11.9214 & 0.7030 & 7.6115 & 6.7280 \\
\hline V1094 Taud & $65.38(7)$ & $70.83(12)$ & $0.2677(4)$ & $5.822(3)$ & $88.21(1)$ & $0.06050(24)$ & $0.04744(29)$ & $5850(100)$ & $5720(100)$ & 13,1 & 23.3292 & 0.9231 & 17.792 & 20.863 \\
\hline CD Tau & $96.8(5)$ & $102.1(5)$ & 0.0 & 0.0 & $87.7(3)$ & $0.1330(10)$ & $0.1172(13)$ & $6200(50)$ & $6194(50)$ & 14 & 13.5172 & 0.9481 & 8.4785 & 9.1246 \\
\hline EW Ori ${ }^{\mathrm{d}}$ & $72.48(21)$ & $75.45(25)$ & $0.076(2)$ & $5.40(2)$ & $89.86(9)$ & $0.05786(18)$ & $0.05434(18)$ & $6070(95)$ & $5875(95)$ & $15,16,17,1$ & 20.2258 & 0.9607 & 18.325 & 18.783 \\
\hline UX Men ${ }^{\mathrm{d}}$ & $87.36(17)$ & $90.08(14)$ & $0.003(3)$ & $1.3(6)$ & $89.6(1)$ & $0.0918(9)$ & $0.0868(9)$ & $6200(100)$ & $6127(100)$ & $18,3,1$ & 14.6652 & 0.9682 & 11.871 & 12.196 \\
\hline TZ Men & $62.15(12)$ & $102.82(45)$ & $0.035(7)$ & $4.75(2)$ & $88.7(1)$ & $0.0722(7)$ & $0.0513(5)$ & $10400(500)$ & $7240(300)$ & 19 & 27.9328 & 0.6045 & 14.480 & 13.018 \\
\hline$\beta$ Aur $^{\mathrm{d}}$ & $108.04(10)$ & $110.93(10)$ & $0.0018(4)$ & $1.579(5)$ & $76.8(1)$ & $0.15694(81)$ & $0.14595(82)$ & $9350(200)$ & $9297(200)$ & $20,21,22,23,1$ & 17.6051 & 0.9739 & 7.3643 & 7.7040 \\
\hline RR Lyn & $65.65(6)$ & 83.92(17) & $0.079(1)$ & $3.14(1)$ & $87.5(1)$ & $0.0878(5)$ & $0.0541(11)$ & $7570(120)$ & $6980(100)$ & 24,25 & 29.340 & 0.7823 & 12.244 & 15.657 \\
\hline WW Aur ${ }^{\mathrm{a}}$ & $116.81(23)$ & $126.49(28)$ & 0.0 & 0.0 & $87.55(4)$ & $0.1586(9)$ & $0.1515(9)$ & $8180(260)$ & $7872(250)$ & 26,1 & 12.1546 & 0.9235 & 7.2450 & 7.1487 \\
\hline HD $71636^{b}$ & $80.30(18)$ & 94.45(19) & 0.0 & 0.0 & $85.63(2)$ & $0.0904(5)$ & $0.0784(5)$ & $6950(140)$ & $6440(140)$ & 27,1 & 17.3682 & 0.8502 & 11.917 & 11.923 \\
\hline VZ Hya & $94.92(19)$ & $105.31(34)$ & 0.0 & 0.0 & $88.88(9)$ & $0.1143(4)$ & $0.0968(6)$ & $6645(150)$ & $6300(150)$ & 28 & 11.4972 & 0.9013 & 9.6587 & 10.367 \\
\hline $\mathrm{KX} \mathrm{Cnc} \mathrm{c}^{\mathrm{b}, \mathrm{a}}$ & $50.039(65)$ & $50.503(65)$ & $0.4667(1)$ & $1.113(1)$ & $89.83(1)$ & $0.01940(4)$ & $0.01913(5)$ & $6050(110)$ & $5995(110)$ & 29,1 & 54.8787 & 0.9908 & 53.405 & 53.674 \\
\hline PT Vel & $117.2(2)$ & $158.5(5)$ & $0.127(6)$ & $5.06(1)$ & $88.2(5)$ & $0.215(2)$ & $0.160(2)$ & $9250(150)$ & $7650(155)$ & 30 & 9.7457 & 0.7394 & 5.5259 & 5.9125 \\
\hline KW Hya ${ }^{d}$ & $70.12(21)$ & 93.17(79) & $0.0945(1)$ & $3.929(2)$ & $87.65(4)$ & $0.0853(5)$ & $0.0594(8)$ & $8000(200)$ & $6960(210)$ & 31,1 & 24.9247 & 0.7526 & 12.559 & 13.908 \\
\hline $\mathrm{RZ} \mathrm{Cha}{ }^{\mathrm{a}}$ & $108.2(6)$ & $107.6(9)$ & 0.0 & 0.0 & $82.89(7)$ & $0.1777(20)$ & $0.1893(40)$ & $6580(150)$ & $6530(150)$ & $32,33,1$ & 12.1746 & 1.0056 & 6.6545 & 6.3340 \\
\hline FM Leo & $76.62(27)$ & $78.46(28)$ & 0.0 & 0.0 & $87.98(6)$ & $0.0798(21)$ & $0.0732(24)$ & $6316(240)$ & $6190(210)$ & 34 & 20.6392 & 0.9765 & 13.505 & 14.354 \\
\hline GG Lup ${ }^{\mathrm{d}}$ & $125.1(5)$ & $203.4(8)$ & $0.154(5)$ & $2.351(3)$ & $86.8(1)$ & $0.2003(18)$ & $0.1456(14)$ & $14750(450)$ & $11200(500)$ & $35,36,1$ & 11.8871 & 0.6150 & 5.7696 & 5.6076 \\
\hline V335 Ser & $106.57(12)$ & $120.07(38)$ & $0.141(2)$ & $1.139(7)$ & $87.2(2)$ & $0.1325(17)$ & $0.1131(22)$ & $9020(150)$ & $8500(150)$ & 37 & 15.3191 & 0.8876 & 8.5918 & 9.0764 \\
\hline $\mathrm{WZ} O \mathrm{Oph}^{\mathrm{a}}$ & $88.77(19)$ & $89.26(24)$ & 0.0 & 0.0 & 89.1(1) & $0.0952(8)$ & $0.0964(8)$ & $6232(100)$ & $6212(100)$ & 28,1 & 14.7240 & 0.9945 & 11.505 & 11.326 \\
\hline FL Lyr $^{\mathrm{d}}$ & $93.5(5)$ & $118.9(7)$ & 0.0 & 0.0 & $86.3(4)$ & $0.140(3)$ & $0.105(3)$ & $6150(120)$ & $5270(110)$ & 15,1 & 9.1640 & 0.7864 & 7.9411 & 8.6028 \\
\hline UZ Dra ${ }^{a}$ & $93.52(35)$ & $101.55(43)$ & 0.0 & 0.0 & $89.1(2)$ & $0.103(2)$ & $0.091(2)$ & $6450(120)$ & $6170(120)$ & $38,39,1$ & 12.5768 & 0.9209 & 10.637 & 11.165 \\
\hline V4089 Sgr $^{\mathrm{b}}$ & $78.48(18)$ & $126.20(24)$ & 0.0 & 0.0 & $83.48(6)$ & $0.2104(9)$ & $0.0852(3)$ & $8433(100)$ & 7361(105) & 40,1 & 18.8426 & 0.6219 & 5.3991 & 8.4925 \\
\hline
\end{tabular}


Table 2

(Continued)

\begin{tabular}{|c|c|c|c|c|c|c|c|c|c|c|c|c|c|c|}
\hline \multirow{3}{*}{$\begin{array}{l}\text { Eclipsing } \\
\text { Binary }\end{array}$} & \multicolumn{9}{|c|}{ Input Parameters } & \multirow{3}{*}{ Reference } & \multicolumn{4}{|c|}{ Model Parameters } \\
\hline & \multicolumn{2}{|c|}{ RV Semiamplitude } & \multicolumn{3}{|c|}{ Orientation of the Orbit } & \multicolumn{2}{|c|}{ Fractional Radius } & \multicolumn{2}{|c|}{ Effective Temperature } & & \multirow{2}{*}{$\begin{array}{c}\text { Semimajor } \\
\text { Axis }\left(R_{\odot}\right)\end{array}$} & \multirow{2}{*}{$\begin{array}{l}\text { Mass } \\
\text { Ratio }\end{array}$} & \multirow{2}{*}{$\Omega_{1}$} & \multirow{2}{*}{$\Omega_{2}$} \\
\hline & $K_{1}\left(\mathrm{~km} \mathrm{~s}^{-1}\right)$ & $K_{2}\left(\mathrm{~km} \mathrm{~s}^{-1}\right)$ & $e$ & $\omega(\mathrm{rad})$ & $i(\operatorname{deg})$ & $r_{1}$ & $r_{2}$ & $T_{1}(\mathrm{~K})$ & $T_{2}(\mathrm{~K})$ & & & & & \\
\hline V1143 Cyg & $88.02(5)$ & $89.97(10)$ & $0.5378(3)$ & $0.860(1)$ & $87.0(1)$ & $0.059(1)$ & $0.058(1)$ & $6450(100)$ & $6400(100)$ & 41,42 & 22.6950 & 0.9783 & 19.069 & 19.045 \\
\hline MY Cyg & $101.9(8)$ & $103.3(6)$ & $0.010(1)$ & $1.21(4)$ & $88.58(2)$ & $0.138(3)$ & $0.134(3)$ & $7050(200)$ & $7000(200)$ & $43,44,45$ & 16.2482 & 0.9867 & 8.2580 & 8.3912 \\
\hline EI Cep ${ }^{\mathrm{d}}$ & $76.84(13)$ & $81.02(13)$ & 0.0 & 0.0 & $87.23(9)$ & $0.1099(20)$ & $0.0884(18)$ & $6750(120)$ & $6977(120)$ & 46,1 & 26.3649 & 0.9484 & 10.056 & 11.760 \\
\hline VZ Cep ${ }^{\mathrm{d}}$ & $118.88(22)$ & $150.48(67)$ & 0.0 & 0.0 & $79.97(5)$ & $0.2398(17)$ & $0.1630(61)$ & $6690(160)$ & $5705(120)$ & 47,1 & 6.3985 & 0.7900 & 4.9955 & 5.9680 \\
\hline LL Aqr & $49.948(13)$ & $57.736(14)$ & $0.3165(1)$ & $2.714(1)$ & $89.55(3)$ & $0.03246(5)$ & $0.02459(8)$ & $6080(50)$ & $5705(60)$ & 48,49 & 40.7438 & 0.8651 & 32.074 & 36.708 \\
\hline EF Aqr ${ }^{b}$ & $84.175(66)$ & $110.66(24)$ & 0.0 & 0.0 & $88.45(8)$ & $0.1222(9)$ & $0.0876(7)$ & $6150(65)$ & $5185(110)$ & 50,1 & 10.9940 & 0.7607 & 8.9529 & 9.8076 \\
\hline V821 Cas ${ }^{\mathrm{b}}$ & $120.8(1.7)$ & $152.4(2.0)$ & $0.127(7)$ & 2.71(7) & $82.6(1)$ & $0.2434(13)$ & $0.1466(17)$ & $9400(400)$ & $8600(400)$ & 51,1 & 9.5600 & 0.7927 & 5.0538 & 6.6690 \\
\hline
\end{tabular}

Notes.

${ }^{\mathrm{a}}$ We recalculated both temperatures-see Section 3.3.

${ }^{\mathrm{b}}$ We recalculated the radial velocity semiamplitudes—-see Section 3.4.

${ }^{\mathrm{c}}$ We set the rotation parameter $F_{1}=18.0$.

${ }^{\mathrm{d}}$ We adjusted the temperature $T_{2}$.

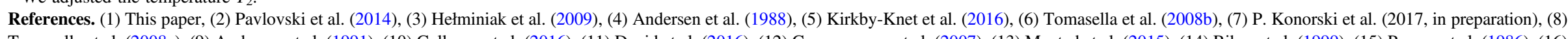

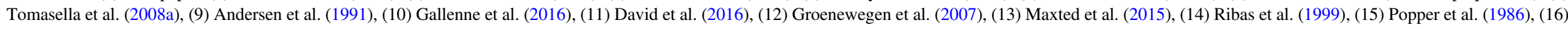

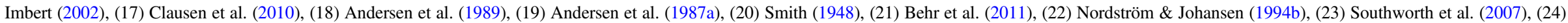

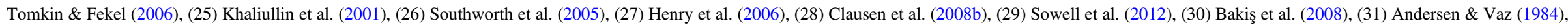

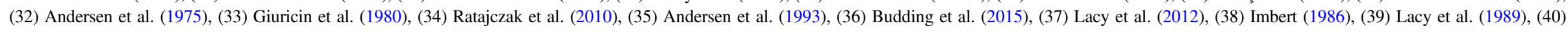

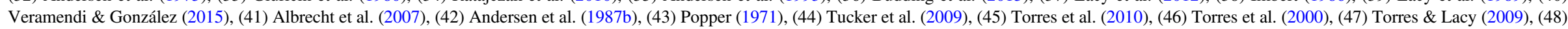
Southworth (2013), (49) Graczyk et al. (2016), (50) Vos et al. (2012), (51) Çakirli et al. (2009). 
Table 3

Photometric and Physical Parameters Used to Derive Individual Angular Diameters and Colors

\begin{tabular}{|c|c|c|c|c|c|c|c|c|c|c|c|c|c|c|c|c|c|}
\hline \multirow{2}{*}{$\begin{array}{l}\text { Eclipsing } \\
\text { Binary }\end{array}$} & \multirow{2}{*}{$\begin{array}{c}E(B-V) \\
(\mathrm{mag})\end{array}$} & \multirow{2}{*}{ References } & \multicolumn{2}{|c|}{ Radius $\left(R_{\odot}\right)$} & \multicolumn{2}{|c|}{ Distance (pc) } & \multirow[t]{2}{*}{$\sigma$} & \multicolumn{5}{|c|}{ Unreddened Johnson Photometry ${ }^{\mathrm{a}}$ (mag) } & \multicolumn{5}{|c|}{ Light ratio $L_{2} / L_{1}{ }^{\mathrm{b}}$} \\
\hline & & & $R_{1}$ & $R_{2}$ & Geom. & Photom. ${ }^{\mathrm{c}}$ & & $B_{0}$ & $V_{0}$ & $J_{0}$ & $H_{0}$ & $K_{0}$ & $B$ & $V$ & $J$ & $H$ & $K$ \\
\hline YZ Cas & $0.015(10)$ & 1 & $2.526(11)$ & $1.332(6)$ & 97.1(4.6) & $99.2(4.0)$ & 0.35 & $5.657(48)$ & $5.607(34)$ & $5.616(21)$ & $5.652(42)$ & $5.635(22)$ & 0.0610 & 0.0882 & 0.1682 & 0.2004 & 0.2046 \\
\hline AI Phe & $0.012(10)$ & 2,1 & $1.841(17)$ & $2.907(13)$ & $168.4(6.8)$ & $167.9(6.7)$ & 0.15 & $9.212(52)$ & $8.573(36)$ & $7.345(25)$ & $6.930(38)$ & $6.832(27)$ & 0.7382 & 1.0057 & 1.6394 & 1.9685 & 1.9828 \\
\hline V505 Per & $0.003(5)$ & 1 & $1.288(14)$ & $1.267(14)$ & $64.3(1.3)$ & $60.7(9)$ & 2.23 & $7.287(34)$ & $6.880(22)$ & $6.117(70)$ & $5.791(40)$ & $5.794(21)$ & 0.9244 & 0.9348 & 0.9522 & 0.9584 & 0.9588 \\
\hline AL Ari & $0.012(10)$ & 3 & $1.375(6)$ & $0.903(4)$ & $140.6(7.3)$ & $137.0(4.0)$ & 0.45 & $9.696(69)$ & $9.186(46)$ & $8.235(23)$ & $7.933(23)$ & $7.905(27)$ & 0.1646 & 0.2100 & 0.3087 & 0.3571 & 0.3597 \\
\hline V570 Per & $0.070(30)$ & 1 & $1.525(30)$ & $1.390(19)$ & $127.4(4.2)$ & $118.6(5.4)$ & 1.29 & $8.270(126)$ & $7.875(94)$ & $7.156(35)$ & $6.921(23)$ & $6.888(22)$ & 0.6567 & 0.6950 & 0.7645 & 0.7916 & 0.7926 \\
\hline TZ For & $0.015(5)$ & 4 & $3.972(84)$ & $8.34(11)$ & $185.9(1.9)$ & $185.2(3.8)$ & 0.17 & $7.569(34)$ & $6.842(22)$ & $5.530(21)$ & $5.124(26)$ & $5.007(30)$ & 0.7888 & 1.2341 & 2.4644 & 3.1856 & 3.2193 \\
\hline V1229 Tau & $0.020(10)$ & 5,6 & $1.729(27)$ & $1.505(45)$ & $132.1(7.0)$ & $133.1(7.5)$ & 0.10 & $6.784(50)$ & $6.745(35)$ & $6.663(25)$ & $6.644(29)$ & $6.637(25)$ & 0.2628 & 0.3385 & 0.5223 & 0.5800 & 0.5917 \\
\hline V1094 Tau & $0.026(10)$ & 7 & $1.411(6)$ & $1.107(7)$ & $121.1(3.7)$ & $118.0(3.8)$ & 0.58 & $9.575(66)$ & $8.901(44)$ & $7.814(23)$ & $7.520(46)$ & $7.437(22)$ & 0.5318 & 0.5524 & 0.5851 & 0.5980 & 0.5986 \\
\hline CD Tau & $0.005(5)$ & 8,1 & $1.798(17)$ & $1.584(20)$ & $73.7(2.1)$ & $68.6(1.2)$ & 2.22 & $7.231(34)$ & $6.753(22)$ & $5.894(22)$ & $5.671(34)$ & $5.612(30)$ & 0.7702 & 0.7724 & 0.7749 & 0.7764 & 0.7760 \\
\hline EW Ori & $0.026(14)$ & 9 & $1.170(5)$ & $1.099(5)$ & $182.5(7.7)$ & $173.8(6.3)$ & 0.88 & $10.407(94)$ & $9.822(61)$ & $8.837(26)$ & $8.598(69)$ & $8.513(22)$ & 0.7209 & 0.7595 & 0.8223 & 0.8474 & 0.8486 \\
\hline UX Men & $0.027(10)$ & 10 & $1.346(13)$ & $1.273(13)$ & $102.9(2.2)$ & $100.6(2.9)$ & 0.62 & $8.692(50)$ & $8.168(35)$ & $7.222(29)$ & $6.966(30)$ & $6.931(25)$ & 0.8318 & 0.8476 & 0.8721 & 0.8818 & 0.8822 \\
\hline TZ Men & $0.000(5)$ & 11 & $2.017(20)$ & $1.433(15)$ & $124.7(7.6)$ & $117.7(8.2)$ & 0.63 & $6.166(34)$ & $6.186(22)$ & $6.180(31)$ & $6.128(44)$ & $6.153(27)$ & 0.1150 & 0.1623 & 0.3002 & 0.3508 & 0.3607 \\
\hline beta Aur & $0.000(3)$ & 1 & $2.763(15)$ & $2.569(15)$ & $24.9(1)$ & $25.0(9)$ & 0.26 & $1.930(34)$ & $1.900(22)$ & $1.869(42)$ & $\ldots$ & $1.859(41)$ & 0.8462 & 0.8524 & 0.8585 & 0.8597 & 0.8603 \\
\hline RR Lyn & $0.007(5)$ & 1 & $2.576(20)$ & $1.587(30)$ & $75.0(3.4)$ & $72.6(2.0)$ & 0.50 & $5.764(33)$ & $5.536(22)$ & & $5.073(23)$ & $5.021(17)$ & 0.2343 & 0.2645 & 0.3298 & 0.3534 & 0.3541 \\
\hline WW Aur & $0.008(5)$ & 1 & $1.928(11)$ & $1.841(11)$ & $90.7(4.1)$ & $85.8(4.0)$ & 1.02 & $5.976(34)$ & $5.807(22)$ & $5.533(22)$ & $5.505(29)$ & $5.513(22)$ & 0.7600 & 0.7953 & 0.8607 & 0.8753 & 0.8789 \\
\hline HD 71636 & $0.020(10)$ & 12 & $1.570(9)$ & $1.362(7)$ & $119.0(5.7)$ & $118.6(4.1)$ & 0.07 & $8.223(51)$ & 7.841(36) & $7.104(22)$ & $6.917(24)$ & $6.907(35)$ & 0.4910 & 0.5442 & 0.6474 & 0.6886 & 0.6911 \\
\hline VZ Hya & $0.027(20)$ & 13 & $1.314(5)$ & $1.113(7)$ & $144.1(5.0)$ & $146.0(7.3)$ & 0.23 & $9.307(93)$ & $8.870(67)$ & $8.105(29)$ & $7.844(28)$ & $7.801(18)$ & 0.5254 & 0.5683 & 0.6439 & 0.6747 & 0.6766 \\
\hline KX Cnc & $0.001(5)$ & 1 & $1.065(2)$ & $1.050(3)$ & $48.7(9)$ & $49.0(1.4)$ & 0.19 & $7.766(35)$ & $7.189(23)$ & $6.223(32)$ & $5.949(30)$ & $5.905(31)$ & 0.9196 & 0.9330 & 0.9537 & 0.9616 & 0.9621 \\
\hline PT Vel & $0.005(5)$ & 14 & $2.095(20)$ & $1.559(20)$ & $163(12)$ & $164.8(4.9)$ & 0.19 & $7.062(34)$ & $7.012(22)$ & $6.902(32)$ & $6.861(32)$ & $6.871(30)$ & 0.2395 & 0.2949 & 0.4199 & 0.4580 & 0.4641 \\
\hline KW Hya & $0.006(6)$ & 1 & $2.126(15)$ & $1.480(22)$ & $86.7(3.2)$ & $86.5(3.6)$ & 0.05 & $6.308(37)$ & $6.081(24)$ & $5.694(24)$ & $5.642(44)$ & $5.574(22)$ & 0.2249 & 0.2699 & 0.3816 & 0.4221 & 0.4255 \\
\hline RZ Cha & $0.038(20)$ & 1 & $2.163(20)$ & $2.305(20)$ & $176.1(8.1)$ & $179.4(8.0)$ & 0.37 & $8.384(87)$ & 7.974(64) & $7.148(35)$ & $6.926(40)$ & $6.919(39)$ & 1.0879 & 1.0988 & 1.1177 & 1.1241 & 1.1250 \\
\hline FM Leo & $0.019(10)$ & 1 & $1.648(43)$ & $1.511(49)$ & $142.9(6.5)$ & $139.6(8.8)$ & 0.27 & $8.884(53)$ & $8.401(37)$ & $7.554(23)$ & $7.324(56)$ & $7.229(24)$ & 0.7441 & 0.7681 & 0.8062 & 0.8216 & 0.8222 \\
\hline GG Lup & $0.027(10)$ & 15 & $2.381(22)$ & $1.732(17)$ & $167.8(8.4)$ & $147.3(9.5)$ & 1.61 & $5.386(48)$ & $5.520(34)$ & $5.861(32)$ & $5.980(38)$ & $5.961(31)$ & 0.3026 & 0.3236 & 0.3774 & 0.3875 & 0.3964 \\
\hline V335 Ser & $0.068(8)$ & 16 & $2.030(26)$ & $1.733(34)$ & 211(13) & $195.2(6.0)$ & 1.15 & $7.352(43)$ & $7.280(30)$ & $7.127(23)$ & $7.105(36)$ & $7.080(22)$ & 0.5730 & 0.6109 & 0.6720 & 0.6870 & 0.6901 \\
\hline wZ Oph & $0.030(16)$ & 1 & $1.402(12)$ & $1.420(12)$ & $151.3(5.5)$ & $164.9(5.7)$ & 1.72 & $9.543(76)$ & $9.033(55)$ & $8.208(34)$ & $7.972(37)$ & $7.894(31)$ & 1.0068 & 1.0115 & 1.0188 & 1.0214 & 1.0217 \\
\hline FL Lyr & $.010(7)$ & 1 & $1.283(30)$ & $0.962(30)$ & $137.9(4.2)$ & $131.9(5.2)$ & 0.83 & $9.875(52)$ & $9.335(34)$ & $8.285(28)$ & 7.983(36) & $7.917(21)$ & 0.2065 & 0.2646 & 0.3969 & 0.4623 & 0.4661 \\
\hline UZ Dra & $0.012(7)$ & 17 & $1.295(25)$ & $1.144(25)$ & $191.9(9.2)$ & $189.7(6.6)$ & 0.20 & $10.036(54)$ & $9.564(35)$ & $8.653(22)$ & $8.423(22)$ & $8.393(20)$ & 0.5991 & 0.6411 & 0.7124 & 0.7419 & 0.7431 \\
\hline V4089 Sgr & $0.027(15)$ & 1 & $3.964(20)$ & $1.605(7)$ & 148(11) & $145.5(4.3)$ & 0.19 & $5.889(67)$ & $5.824(49)$ & $5.710(25)$ & $5.627(40)$ & $5.623(25)$ & 0.0776 & 0.0953 & 0.1327 & 0.1452 & 0.1466 \\
\hline V1143 Cyg & $0.000(5)$ & 18,1 & $1.339(23)$ & $1.316(23)$ & $40.4(6)$ & $39.2(1.1)$ & 1.02 & $6.347(34)$ & $5.889(22)$ & $5.024(21)$ & $4.845(22)$ & $4.798(21)$ & 0.9233 & 0.9339 & 0.9512 & 0.9577 & 0.9581 \\
\hline MY Cyg & $0.048(30)$ & 19 & $2.242(50)$ & $2.178(50)$ & $253(15)$ & $229(14)$ & 1.17 & $8.520(126)$ & $8.193(94)$ & $7.702(51)$ & $7.563(46)$ & $7.538(26)$ & 0.9075 & 0.9158 & 0.9312 & 0.9360 & 0.9363 \\
\hline EI Cep & $0.007(5)$ & 20 & $2.898(48)$ & $2.331(44)$ & 197.2(9.3) & $194.0(5.8)$ & 0.30 & $7.956(35)$ & $7.578(23)$ & $6.879(21)$ & $6.738(37)$ & $6.696(18)$ & 0.7702 & 0.7405 & 0.6891 & 0.6742 & 0.6723 \\
\hline VZ Cep & $0.044(10)$ & 21 & $1.534(12)$ & $1.043(39)$ & $258(23)$ & $211.1(8.5)$ & 2.07 & $10.025(46)$ & $9.581(32)$ & $8.796(23)$ & $8.621(33)$ & $8.606(22)$ & 0.1764 & 0.2256 & 0.3308 & 0.3830 & 0.3851 \\
\hline LL Aqr & $0.018(14)$ & 22 & $1.323(6)$ & $1.002(5)$ & $129.0(4.5)$ & $132.4(3.6)$ & 0.57 & $9.748(86)$ & $9.187(57)$ & $8.180(26)$ & $7.864(37)$ & $7.836(24)$ & 0.3848 & 0.4268 & 0.4998 & 0.5306 & 0.5320 \\
\hline EF Aqr & $0.025(15)$ & 23 & $1.343(10)$ & $0.963(8)$ & 198(20) & $169.4(6.0)$ & 1.57 & $10.385(77)$ & $9.808(51)$ & $8.849(27)$ & $8.511(29)$ & $8.496(24)$ & 0.1678 & 0.2207 & 0.3480 & 0.4141 & 0.4174 \\
\hline V821 Cas & $0.060(30)$ & 1 & $2.327(29)$ & $1.401(33)$ & $277(23)$ & $306(24)$ & 0.91 & $8.158(125)$ & $8.101(94)$ & $7.976(29)$ & 7.994(32) & $7.975(29)$ & 0.2554 & 0.2829 & 0.3231 & 0.3333 & 0.3354 \\
\hline
\end{tabular}

Notes.

${ }^{\text {a }}$ Combined extinction-corrected out-of-eclipse magnitudes of both components expressed in Johnson photometric system.

${ }^{\mathrm{b}}$ Calculated using the WD model.

${ }^{\mathrm{c}}$ Photometric distances derived from bolometric flux scaling.

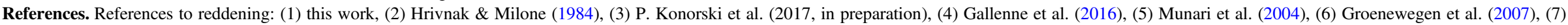

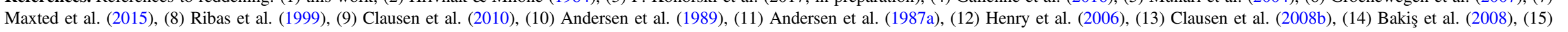

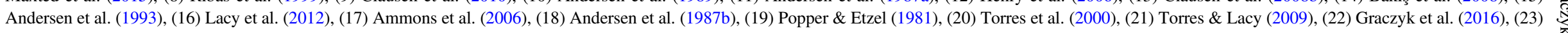
Vos et al. (2012), 
described below. The rotation parameter $F_{1,2}$ was kept to 1 (synchronous rotation), unless there was a direct spectroscopic determination of $F$ significantly different from unity. The albedo $A$ and the gravity brightening $g$ were set in a standard way for a convective atmosphere cooler than $7200 \mathrm{~K}$ and for radiative atmospheres for a hotter surface temperature. This was done only for the sake of consistency because the two parameters have negligible effect on the light ratios. The input and derived parameters used to create the appropriate WD models are listed in Table 2.

\subsection{Correction of 2MASS Magnitudes Taken During Eclipses}

The systems KX Cnc, GG Lup, and WW Oph have 2MASS observations taken during the secondary eclipses. To account for light lost during minima, we used our models to calculate the appropriate corrections. The corrections are $\Delta J=-0.333 \mathrm{mag}$, $\Delta H=-0.331 \mathrm{mag}$, and $\Delta K=-0.331 \mathrm{mag}$ for $\mathrm{KX}$ Cnc, $\Delta J=$ $-0.277 \mathrm{mag}, \Delta H=-0.281 \mathrm{mag}$, and $\Delta K=-0.285 \mathrm{mag}$ for GG Lup, $\Delta J=-0.392$ mag, $\Delta H=-0.390 \mathrm{mag}$, and $\Delta K=-0.390 \mathrm{mag}$ for WZ Oph. For GG Lup we also accounted for the apsidal motion that shifts the position of the eclipses (Wolf \& Zejda 2005).

\subsection{Temperature and Reddening}

In some individual cases, described in the Appendix, the temperature $T_{1}$ or/and color excess $E(B-V)$ were adjusted in order to obtain agreement between intrinsic colors and temperatures. Reddenings to each object were taken from the literature, when available, and were also derived independently using the extinction maps by Schlegel et al. (1998) following the prescription given in Suchomska et al. (2015). Dereddened magnitudes and colors were calculated using the mean Galactic interstellar extinction curve from Fitzpatrick \& Massa (2007) assuming $R_{V}=3.1$. To rederive temperatures, we used a number of calibrations given below:

1. $b-y$ : Holmberg et al. (2007), Ramírez \& Meléndez (2005), Alonso et al. (1996), and Napiwotzki et al. (1993).

2. $B-V$ : Casagrande et al. (2010), González Hernández \& Bonifacio (2009), Ramírez \& Meléndez (2005), and Flower (1996).

3. V-J: Casagrande et al. (2010) and González Hernández \& Bonifacio (2009).

4. $V-K$ : Worthey \& Lee (2011), Casagrande et al. (2010), González Hernández \& Bonifacio (2009), Masana et al. (2006), Ramírez \& Meléndez (2005), Houdashelt et al. (2000), and Alonso et al. (1996).

\subsection{Radial Velocity Semiamplitudes}

We typically assumed radial velocity semiamplitudes from the literature. When two or more orbital solutions were published based on different radial velocity sets and with uncertainties on the same order of magnitude, we used the weighted mean to derive the final parameters. This was the case for AI Phe, EW Ori, UX Men, $\beta$ Aur, GG Lup, UZ Dra, and V1143 Cyg. However, in a few cases, we redetermined the spectroscopic orbits from source data in order to directly derive $K_{1,2}$ or to check the consistency of the orbital parameters and their errors. The spectroscopic orbits were derived with the Wilson-Devinney code taking into account the full model of a system and all proximity effects. A set of numerical constants used to change from SI units into astrophysical units were chosen according to the prescription of Torres et al. (2010). Individual cases are described in the Appendix.

\subsection{Distances}

\subsubsection{Geometric Distances}

The source of parallaxes is almost exclusively the recent release of Gaia parallaxes DR1 (Gaia Collaboration et al. 2016), and in a few cases of close and bright systems where these parallaxes are unavailable, we use parallaxes from the latest reduction of the Hipparcos data (van Leeuwen 2007). Distances are calculated through simple inversion of trigonometric parallaxes. It is known that this procedure for larger parallax errors $(24 \%)$ is not unequivocal and must include some prior on expected space distribution of an object (e.g., Sandage \& Saha 2002; Bailer-Jones 2015). Existence of this prior is necessary to recover a true distribution (distances) from an observed distribution (parallaxes) in the presence of observational errors. In terms of absolute luminosity bias, it leads to the so-called Lutz-Kelker correction (Lutz \& Kelker 1973). However, errors given by the Gaia DR1 are preliminary and likely overestimated (e.g., Casertano et al. 2016), and using them for parallax corrections would introduce unknown amounts of systematics. For the purpose of this paper, we decided to not apply Lutz-Kelker corrections to the distances, especially as any such correction would be smaller than the quoted errors. The resulting distances are summarized in Table 3.

\subsubsection{Photometric Distances}

We employed the so-called standard method using $V$-band bolometric corrections to derive photometric distances, known also as the bolometric flux scaling. We calculated the distance $d$ to the $i$ th component of the system using the equation

$$
d_{i}(\mathrm{pc})=3.360 \times 10^{-8} R_{i} T_{i}^{2} 10^{0.2\left(B C_{i}+V_{i}\right)},
$$

where the index $i=\{1,2\}, R$ is the radius of a component in solar radii, $T$ is its effective temperature in $\mathrm{K}, B C$ is a bolometric correction interpolated from the Flower (1996) tables for a given temperature, and $V$ is the intrinsic magnitude of a component (corrected for extinction). The distance to a particular system was calculated as the unweighted average distance of the two components. The purpose of introducing photometric distances is to check for consistency of the eclipsing binary model parameters and to validate the Gaia parallaxes used in the analysis. The photometric distances are given in Table 3.

\subsection{Angular Diameters}

In order to derive SBC relations, we need to calculate individual angular diameters of the stars. Angular diameters are calculated with the formula

$$
\phi(\mathrm{mas})=9.3004 \cdot R\left(R_{\odot}\right) / d(\mathrm{pc}),
$$

where $d$ is the distance, $R$ is the radius of the star, and the conversion factor is equal to $2000 R_{\odot} / 1$ au assuming a solar radius $R_{\odot}=695,660 \mathrm{~km}$ (Habbereiter et al. 2008) and the 
length of the astronomical unit $1 \mathrm{au}=149,597,871 \mathrm{~km}$ (Pitjeva \& Standish 2009).

We emphasize that the angular diameter calculated from the photometric distance is a function of radiative properties of a star (mainly its effective temperature) and not its geometric properties. Indeed, when we combine Equations (1) and (2), we derive angular diameter that is only a function of the effective temperature, the $V$-band bolometric correction (also temperature dependent), and extinction-corrected $V$-band magnitude. Because of this, we do not use the photometric distances to calculate angular diameters in the present work.

\subsection{Intrinsic Magnitudes}

In Table 3 we summarize all parameters used to derive the intrinsic photometric indexes of components. The mean galactic extinction curve with $R_{V}=3.1$ (Fitzpatrick \& Massa 2007) was used to correct the observed magnitudes for reddening. Next, with the help of our WD models, we calculated light ratios in the Johnson $B V J H K$ bands and used them to derive intrinsic magnitudes and colors of each component. The WD code uses an atmospheric approximation with intensities based on ATLAS9 (Kurucz 1993, p. 13) model stellar atmospheres, which are integrated over a given passband to give the emerging flux, which is expressed as the fraction of flux that emerges from the blackbody of the same temperature. For all the systems in our sample, $B$ and $V$ light ratios are tuned to published light ratios based on literature light-curve solutions. However, in order to calculate the light ratios in the infrared $J H K$ bands, we need to extrapolate the models as none of the systems has published or analyzed infrared light curves. This is why the temperature ratio needs to be well established in order to minimize systematics that are due to the extrapolation. When the temperature ratio and absolute temperatures are well known, this procedure does not introduce significant bias because the relative fluxes from the atmospheric models are much better constrained than the absolute fluxes. We add also that errors given on unreddened magnitudes in Table 3 do not account for possible systematic shifts on a level of $1 \%$ that are due to the transformation of Tycho-2 and 2MASS magnitudes into the Johnson photometric system.

\subsection{Surface Brightness}

We follow Hindsley \& Bell (1989) to define the surface brightness parameter $S$ :

$$
S_{i}=m_{i, 0}+5 \log \phi
$$

where $i$ denotes a particular band ( $B$ or $V$ ), and $m_{i, 0}$ is the intrinsic magnitude in a given band. The surface brightness parameter $S$ was then used to obtain the SBC relations by fitting it with first- and fifth-degree polynomials in the form

$$
S=\sum_{i=0}^{i=1,5} a_{i} X^{i}
$$

where $X$ is a given photometric color (see Section 4.3 for more details). Use of a higher-order polynomial is justified by the strong nonlinearity of the SBC relations for the bluest colors (stellar spectral types earlier than A0).

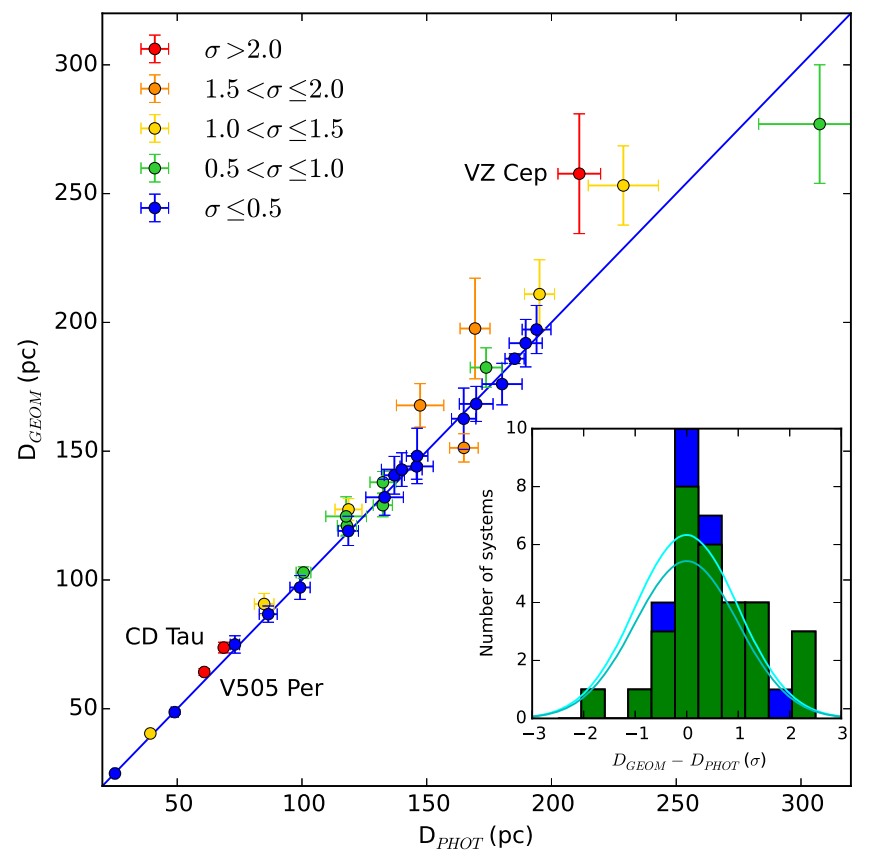

Figure 1. Comparison of geometric and photometric distances for all systems with deviation from a 1:1 relation expressed as fraction of $\sigma$ and coded with color. Three named systems exhibit offsets larger than $2 \sigma$. Systems with $\sigma \leqslant 0.5$ define the best-fit subsample. Inset: the expected distribution of deviations from a 1:1 relation for all systems (upper line) and systems with the Gaia parallaxes (lower line) when random errors dominate. The histogram shows the actual distribution of deviations for the Gaia subsample (green) and the entire sample (green+blue).

\section{Results}

\subsection{Distances}

Stassun \& Torres (2016a, 2016b) presented comprehensive comparisons of geometric distances from the Hipparcos and Gaia satellites with photometric distances derived from eclipsing binaries. We underline that we use here a different method to derive photometric distances, and while our sample is significantly smaller, it is more carefully selected. Figure 1 shows the comparison of geometric and photometric distances, where the difference between the two distances is expressed in terms of the standard deviation of distances $\sigma$. Inspection of the figure confirms that detached eclipsing binary stars serve as almost perfect distance indicators, and the photometric distance is a very good proxy of the true geometric distance, as long as issues with reddening and temperature are properly handled (e.g., see Section 4 in Torres et al. 2010). The distances and their $\sigma$ difference are given in Table 3 .

The largest deviations from the 1:1 relation between geometric and photometric distance are found for V505 Per $(2.2 \sigma)$, CD Tau $(2.2 \sigma)$, and $\mathrm{VZ} \operatorname{Cep}(2.1 \sigma)$. For the complete sample the reduced $\chi^{2}=0.99$ (34 degrees of freedom) i.e., it is fully consistent with statistical uncertainties dominating the error budget in the distance determination. However, when we exclude the three most strongly deviating systems, the reduced $\chi^{2}=0.87$ (31 degrees of freedom) suggests that for the majority of systems, the errors on the distance determinations are slightly overestimated. We note that the two strongly deviating systems (CD Tau and VZCep) also stand out in a comparison of photometric and Hipparcos parallaxes (Stassun \& Torres 2016a). Possible explanations are incorrectly 
estimated temperatures or/and interstellar extinctions for these two systems or some additional systematics in the trigonometric parallaxes. However, more work is needed to determine the source of the discrepancy.

The inset in Figure 1 shows the histogram of the deviations in terms of the $\sigma$ from a subsample with Gaia parallaxes (30 systems, green) and from the remaining sample (green+blue). Superimposed are the expected distributions of deviations when errors are uncorrelated and dominated by random uncertainties. We see a clear excess of systems with small deviations, signifying that errors on distances are inflated for both the sample and the Gaia subsample. This is in agreement with the conclusion by Casertano et al. (2016). We see also that Gaia distances are on average larger than photometric distances, thus corroborating findings by Stassun $\&$ Torres (2016b).

\subsection{Angular Diameters}

The distances were used to calculate geometric angular diameters for the entire sample-see Table 4. These angular diameters are direct limb-darkened angular diameters, and they are complimentary to angular diameters derived from interferometry (e.g., compilations by Boyajian et al. 2014; Challouf et al. 2014). They have an average precision of $4.7 \%$ that is limited by the precision of parallax determinations. The precision is better than $2 \%$ for 11 components. Figure 2 shows the derived angular diameters with uncertainties. We note the clear dependency of the uncertainty on angular size and color $(V-K)_{0}$, with the angular diameters of bluer (hotter) stars more poorly determined. Of the entire sample, only one star, the cooler component of TZ For, had its angular diameter measured directly with interferometry (Gallenne et al. 2016), but with much lower precision, and two components of $\beta$ Aur were barely resolvable (Hummel et al. 1995).

\subsection{SBC Relations}

Figure 3 shows the relation between the $V$-band surface brightness $S_{V}$ and color $(V-K)_{0}$ against some interferometric SBC relations (Kervella et al. 2004; Di Benedetto 2005; Boyajian et al. 2014; Challouf et al. 2014). The left and right panels correspond to $S_{V}$ derived from the complete sample and the best-fit subsample, respectively. The best-fit systems were defined as those whose geometrical and photometric distances agreed to better than $0.5 \sigma$ - see Table 3 and Figure 1 . The $V$-band surface brightnesses derived from trigonometric parallaxes fits the Challouf et al. (2014) calibration well, with a spread of $\sim 0.1 \mathrm{mag}$ corresponding to $5 \%$ uncertainty in angular diameter, dominated by distance errors. The agreement with the interferometric relation is satisfactory, i.e., both methods of measuring angular diameters, direct from interferometry and semidirect from eclipsing binary stars, show good consistency. The agreement is even better if we use the best-fit subsample.

In order to quantify the SBC relation, we derived it directly. We fit Equation (4) to the $S_{V}$ (see Section 3.8) using orthogonal distance regression (Boggs \& Rogers 1990), which accounts for the errors on the independent variable, in our case, color $(V-K)_{0}$. We fit a fifth-order polynomial to all the data and a firstorder polynomial to the data of the best-fit systems. The results of the fitting are presented in Figure 4 and coefficients of the derived relations are given in Table 5. The precision of the SBC relation based on all systems is rather low $(\sim 5 \%)$ with the distance errors fully dominating the error budget. However, the use of systems with the best consistency of their geometric and photometric distances results in a remarkable improvement of the precision of the derived SBC relation by a factor of 2 . The internal precision of the linear relation in predicting angular diameters of A-, F-, and G-type stars is in fact comparable to or even better than currently published interferometric relations, e.g.,: Boyajian et al. (2014) - 4.6\%, Challouf et al. (2014)$3.7 \%$, Kervella et al. (2004) $-2.8 \%{ }^{15}$, and Di Benedetto (2005) $-2.1 \%$. The linear SBC relation we derived is almost indistinguishable from the relation by Boyajian et al. (2014), it compares well with the relation by Challouf et al. (2014), especially for the bluest colors, and also with Di Benedetto (2005) for the reddest colors $(V-K)_{0}>1.0$. This is an important argument in favor of the eclipsing binary method as a fully independent way to derive the SBC calibration.

One of the advantages of using eclipsing binary stars is the very precise surface gravities derived for the individual components. This allows us in principle to determine how a SBC relation might depend on surface gravity (Figure 4). We see some hints of this dependence where higher surface gravities result in higher surface brightness, but the spread is still large and it is premature to draw a conclusion here.

The broadband SBC relations calibrated onto a wide range of colors do not show any statistically significant metallicity dependence, with the exception of the bluest colors (e.g., $(B-V)$, see Boyajian et al. 2014). We compiled the metallicity determinations for our sample from the literature (Table 4) in order to check the possible dependence. As expected, no clear metallicity dependence is visible for the $V-K$ color-see Figure 5, although the scatter may hide it.

For the SBC relation to be useful, it should have small intrinsic scatter and be only weakly dependent on reddening. The SBC relation for the $V$ band and $(V-K)$ has great potential in this respect. This relation is commonly used to predict angular diameters and to determine distances, e.g., to the Magellanic Clouds with an accuracy of 2\%-3\% (Pietrzyński et al. 2013; Graczyk et al. 2014). However, for early-type stars $(\mathrm{O}$ or $\mathrm{B})$, the relation becomes non-negligibly inclined to the line of reddening and shows significantly larger scatter than for stars with spectral types later than A5 (e.g., Challouf et al. 2014). This reduces its potential for predicting angular diameters of early-type stars. Kervella et al. (2004) reported that the SBC relations based on colors with a larger wavelength difference show smaller scatter, i.e., the colors $(B-K)$ and $(V-L)$. However, their relations were constrained to intermediate- and late-type stars.

We decided to search for similar relations using our sample. We combined two surface brightness parameters $(B-$ and $V$-band) with the six colors $(V-J),(V-H),(V-K),(B-J)$, $(B-H)$, and $(B-K)$. We fit the surface brightness parameters for the best-fit and all systems using first- and fifth-order polynomials, respectively. Figure 6 shows the two derived promising SBC relations based on $(B-K)$ color and with the rms minimized. The appropriate polynomial coefficients and the precision of the angular diameter prediction are reported in Table 5. Both relations give precisions in the predicted angular diameters of $5 \%$ for all the stars, $2 \%-3 \%$ for the best-fit subsample, and have the smallest inclination of the relations to

\footnotetext{
15 The precision of $S_{V^{-}}(V-K)$ calibration by Kervella et al. (2004) is reported to be $1 \%$. We recalculated the unweighted root mean square from data given in Kervella et al. (2004). We obtained a relative precision of $\sim 3 \%$, which we quote above.
} 
Table 4

Metallicities from the Literature and Derived Quantities: Masses, Gravities, and Geometric Angular Diameters of All Eclipsing Binary Components

\begin{tabular}{|c|c|c|c|c|c|c|c|c|}
\hline \multirow{2}{*}{ ID } & \multirow{2}{*}[\mathrm{Fe}/\mathrm{H}]{} & \multirow{2}{*}{ References } & \multicolumn{2}{|c|}{ Mass } & \multicolumn{2}{|c|}{ Gravity } & \multicolumn{2}{|c|}{ Angular Diameter } \\
\hline & & & $\begin{array}{c}M_{1} \pm \sigma \\
\left(M_{\odot}\right)\end{array}$ & $\begin{array}{c}M_{2} \pm \sigma \\
\left(M_{\odot}\right)\end{array}$ & $\begin{array}{l}\log g_{1} \pm \sigma \\
\quad(\operatorname{dex})\end{array}$ & $\begin{array}{l}\log g_{2} \pm \sigma \\
\quad(\operatorname{dex})\end{array}$ & $\begin{array}{c}\theta_{1} \pm \sigma \\
\text { (mas) }\end{array}$ & $\begin{array}{c}\theta_{2} \pm \sigma \\
\text { (mas) }\end{array}$ \\
\hline YZ Cas & 0.10 & 1 & $2.263 \pm 0.012$ & $1.325 \pm 0.007$ & $3.988 \pm 0.004$ & $4.311 \pm 0.005$ & $0.242 \pm 0.012$ & $0.128 \pm 0.006$ \\
\hline AI Phe & -0.14 & 2 & $1.193 \pm 0.004$ & $1.242 \pm 0.004$ & $3.985 \pm 0.008$ & $3.605 \pm 0.004$ & $0.102 \pm 0.004$ & $0.161 \pm 0.007$ \\
\hline V505 Per & -0.12 & 3 & $1.272 \pm 0.003$ & $1.254 \pm 0.003$ & $4.323 \pm 0.009$ & $4.331 \pm 0.010$ & $0.186 \pm 0.004$ & $0.183 \pm 0.004$ \\
\hline AL Ari & -0.00 & 4 & $1.170 \pm 0.006$ & $0.916 \pm 0.004$ & $4.230 \pm 0.004$ & $4.489 \pm 0.004$ & $0.091 \pm 0.005$ & $0.060 \pm 0.003$ \\
\hline V570 Per & 0.02 & 5 & $1.452 \pm 0.009$ & $1.352 \pm 0.009$ & $4.234 \pm 0.017$ & $4.283 \pm 0.012$ & $0.111 \pm 0.004$ & $0.101 \pm 0.004$ \\
\hline TZ For & 0.02 & 6 & $1.957 \pm 0.002$ & $2.056 \pm 0.002$ & $3.532 \pm 0.018$ & $2.909 \pm 0.011$ & $0.199 \pm 0.005$ & $0.417 \pm 0.007$ \\
\hline V1229 Tau & 0.06 & 7 & $2.203 \pm 0.013$ & $1.549 \pm 0.010$ & $4.306 \pm 0.014$ & $4.273 \pm 0.026$ & $0.122 \pm 0.007$ & $0.106 \pm 0.006$ \\
\hline V1094 Tau & -0.09 & 8 & $1.096 \pm 0.004$ & $1.012 \pm 0.003$ & $4.179 \pm 0.004$ & $4.355 \pm 0.006$ & $0.108 \pm 0.003$ & $0.085 \pm 0.003$ \\
\hline CD Tau & 0.08 & 9 & $1.441 \pm 0.016$ & $1.367 \pm 0.016$ & $4.087 \pm 0.010$ & $4.174 \pm 0.012$ & $0.227 \pm 0.007$ & $0.200 \pm 0.006$ \\
\hline EW Ori & 0.05 & 10 & $1.177 \pm 0.009$ & $1.130 \pm 0.008$ & $4.373 \pm 0.005$ & $4.409 \pm 0.005$ & $0.060 \pm 0.003$ & $0.056 \pm 0.002$ \\
\hline UX Men & 0.04 & 11 & $1.229 \pm 0.006$ & $1.192 \pm 0.007$ & $4.270 \pm 0.009$ & $4.305 \pm 0.009$ & $0.122 \pm 0.003$ & $0.115 \pm 0.003$ \\
\hline TZ Men & $\cdots$ & & $2.482 \pm 0.025$ & $1.500 \pm 0.010$ & $4.224 \pm 0.010$ & $4.302 \pm 0.010$ & $0.150 \pm 0.009$ & $0.107 \pm 0.007$ \\
\hline beta Aur & 0.15 & 12 & $2.365 \pm 0.006$ & $2.303 \pm 0.006$ & $3.929 \pm 0.005$ & $3.981 \pm 0.005$ & $1.033 \pm 0.008$ & $0.961 \pm 0.008$ \\
\hline RR Lyn & -0.24 & 13 & $1.922 \pm 0.026$ & $1.504 \pm 0.041$ & $3.900 \pm 0.009$ & $4.214 \pm 0.020$ & $0.320 \pm 0.015$ & $0.197 \pm 0.010$ \\
\hline WW Aur & $\cdots$ & & $1.964 \pm 0.010$ & $1.814 \pm 0.008$ & $4.161 \pm 0.005$ & $4.167 \pm 0.006$ & $0.198 \pm 0.009$ & $0.189 \pm 0.009$ \\
\hline HD 71636 & -0.05 & 14 & $1.512 \pm 0.007$ & $1.285 \pm 0.006$ & $4.226 \pm 0.005$ & $4.279 \pm 0.005$ & $0.123 \pm 0.006$ & $0.106 \pm 0.005$ \\
\hline VZ Hya & -0.20 & 15 & $1.271 \pm 0.009$ & $1.146 \pm 0.006$ & $4.305 \pm 0.005$ & $4.404 \pm 0.006$ & $0.085 \pm 0.003$ & $0.072 \pm 0.003$ \\
\hline $\mathrm{KX} \mathrm{Cnc}$ & 0.07 & 16 & $1.142 \pm 0.003$ & $1.132 \pm 0.003$ & $4.441 \pm 0.002$ & $4.450 \pm 0.003$ & $0.203 \pm 0.004$ & $0.201 \pm 0.004$ \\
\hline PT Vel & $\cdots$ & & $2.199 \pm 0.016$ & $1.626 \pm 0.009$ & $4.138 \pm 0.009$ & $4.264 \pm 0.011$ & $0.120 \pm 0.009$ & $0.089 \pm 0.007$ \\
\hline KW Hya & $\cdots$ & & $1.973 \pm 0.036$ & $1.485 \pm 0.017$ & $4.078 \pm 0.010$ & $4.269 \pm 0.014$ & $0.228 \pm 0.008$ & $0.159 \pm 0.006$ \\
\hline RZ Cha & -0.02 & 17 & $1.505 \pm 0.027$ & $1.513 \pm 0.021$ & $3.946 \pm 0.011$ & $3.893 \pm 0.010$ & $0.114 \pm 0.005$ & $0.122 \pm 0.006$ \\
\hline FM Leo & $\cdots$ & & $1.318 \pm 0.011$ & $1.287 \pm 0.010$ & $4.124 \pm 0.023$ & $4.189 \pm 0.028$ & $0.107 \pm 0.006$ & $0.098 \pm 0.006$ \\
\hline GG Lup & -0.10 & 18 & $4.079 \pm 0.039$ & $2.508 \pm 0.022$ & $4.295 \pm 0.009$ & $4.360 \pm 0.009$ & $0.132 \pm 0.007$ & $0.096 \pm 0.005$ \\
\hline V335 Ser & $\cdots$ & & $2.147 \pm 0.014$ & $1.905 \pm 0.008$ & $4.155 \pm 0.011$ & $4.240 \pm 0.017$ & $0.089 \pm 0.006$ & $0.076 \pm 0.005$ \\
\hline WZ Oph & -0.27 & 15 & $1.227 \pm 0.007$ & $1.220 \pm 0.006$ & $4.233 \pm 0.008$ & $4.220 \pm 0.008$ & $0.086 \pm 0.003$ & $0.087 \pm 0.003$ \\
\hline FL Lyr & -0.30 & 19 & $1.218 \pm 0.016$ & $0.958 \pm 0.012$ & $4.307 \pm 0.021$ & $4.453 \pm 0.028$ & $0.087 \pm 0.003$ & $0.065 \pm 0.003$ \\
\hline UZ Dra & $\ldots$ & & $1.306 \pm 0.012$ & $1.203 \pm 0.011$ & $4.330 \pm 0.017$ & $4.402 \pm 0.019$ & $0.063 \pm 0.003$ & $0.055 \pm 0.003$ \\
\hline V4089 Sgr & $\cdots$ & & $2.584 \pm 0.012$ & $1.607 \pm 0.008$ & $3.654 \pm 0.005$ & $4.233 \pm 0.004$ & $0.249 \pm 0.018$ & $0.101 \pm 0.007$ \\
\hline V1143 Cyg & 0.08 & 20 & $1.356 \pm 0.003$ & $1.328 \pm 0.002$ & $4.317 \pm 0.015$ & $4.323 \pm 0.015$ & $0.308 \pm 0.007$ & $0.303 \pm 0.007$ \\
\hline MY Cyg & $\cdots$ & & $1.806 \pm 0.025$ & $1.782 \pm 0.030$ & $3.994 \pm 0.020$ & $4.013 \pm 0.021$ & $0.082 \pm 0.005$ & $0.080 \pm 0.005$ \\
\hline EI Cep & -0.04 & 21 & $1.772 \pm 0.006$ & $1.680 \pm 0.006$ & $3.762 \pm 0.014$ & $3.928 \pm 0.016$ & $0.137 \pm 0.007$ & $0.110 \pm 0.006$ \\
\hline VZ Cep & 0.06 & 22 & $1.402 \pm 0.015$ & $1.108 \pm 0.008$ & $4.213 \pm 0.008$ & $4.446 \pm 0.033$ & $0.055 \pm 0.005$ & $0.038 \pm 0.004$ \\
\hline LL Aqr & 0.02 & 23 & $1.195 \pm 0.001$ & $1.034 \pm 0.001$ & $4.272 \pm 0.004$ & $4.451 \pm 0.004$ & $0.095 \pm 0.003$ & $0.072 \pm 0.003$ \\
\hline EF Aqr & 0.00 & 24 & $1.243 \pm 0.006$ & $0.946 \pm 0.003$ & $4.276 \pm 0.007$ & $4.447 \pm 0.007$ & $0.063 \pm 0.006$ & $0.045 \pm 0.004$ \\
\hline V821 Cas & $\ldots$ & & $2.088 \pm 0.064$ & $1.655 \pm 0.050$ & $4.024 \pm 0.017$ & $4.364 \pm 0.024$ & $0.078 \pm 0.007$ & $0.047 \pm 0.004$ \\
\hline
\end{tabular}

References. References to metallicities: (1) Pavlovski et al. (2014), (2) Andersen et al. (1988), (3) Tomasella et al. (2008b), (4) P. Konorski et al. (2017, in preparation), (5) Tomasella et al. (2008a), (6) Gallenne et al. (2016), (7) Groenewegen et al. (2007), (8) Maxted et al. (2015), (9) Ribas et al. (1999), (10) Clausen et al. (2010), (11) Andersen et al. (1989), (12) Southworth et al. (2007), (13) Khaliullin et al. (2001), (14) Holmberg et al. (2009), (15) Clausen et al. (2008b), (16) Sowell et al. (2012), (17) Jørgensen \& Gyldenkerne (1975), (18) Andersen et al. (1993), (19) Guillout et al. (2009), (20) Andersen et al. (1987b), (21) Torres et al. (2000), (22) Torres \& Lacy (2009), (23) Graczyk et al. (2016), (24) Vos et al. (2012).

the reddening line for bluest colors. We note here that the real precision is lower because of the global systematic uncertainty of Gaia DR1 parallaxes. We estimated that the upper limit of the systematics is about $3 \%$ for our systems.

\section{Discussion}

The main purpose of the paper is to show that the inverse eclipsing binary method allows an independent and precise calibration of the SBC relations. The results presented in Section 4.3 fully corroborate this premise. Still, the precision of the derived relations is not significantly better than the precision derived from interferometric measurements of stellar angular diameters. In this section we quantify the steps required to reach a precision better than $1 \%$ in predicting angular diameters.

\subsection{Uniform Analysis}

We compiled in this work data from numerous papers published by many different groups of researchers. Each group uses different quality photometric and spectroscopic data, 


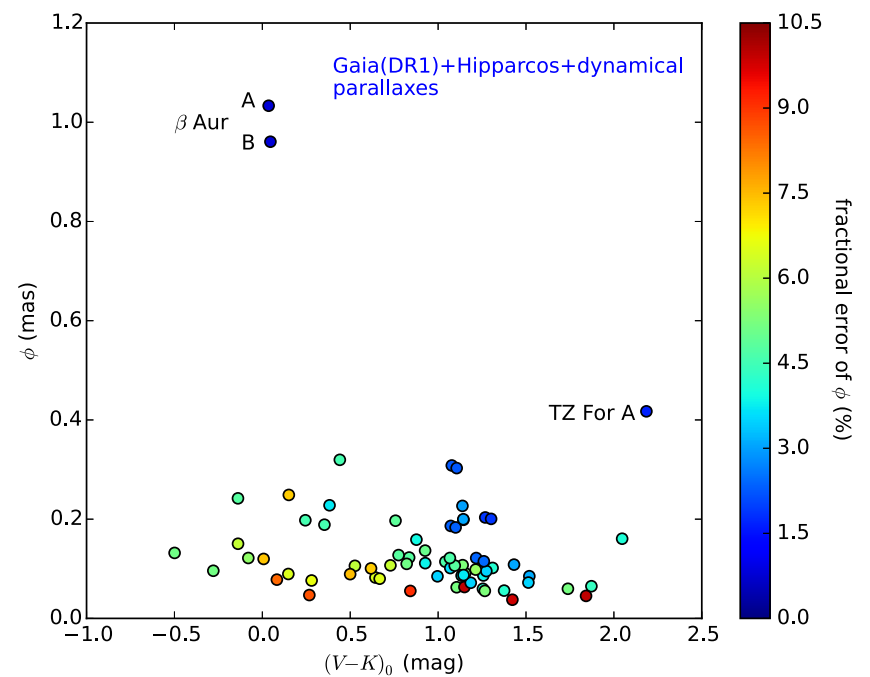

Figure 2. Geometric angular diameters of the eclipsing binary components and their uncertainties calculated from the most recent stellar radii and parallaxes. Three stars that are resolvable by interferometry are named.

different methods to derive radial velocities, different analyses of light curves and radial velocity curves (separated, simultaneous, single light curve, multiband light curves), different ways of deriving effective temperatures (color-temperature calibrations, atmospheric model analysis), and finally different sets of astrophysical numerical constants. During this work, we made some effort to homogenize the existing data on eclipsing binary stars, but it was constrained to the effective temperatures, their ratio, and the radial velocity semiamplitudes.

However, to determine systematics, a full homogenous reanalysis of each system would be needed with the same method and software, and also similar quality observables. This would result in a better evaluation of the relative precision of each data set, and it would augment the internal precision of the physical parameters of the whole sample. Significant help in this respect can be expected from using new high-precision numerical codes such as ellc (Maxted 2016) or Phoebe-2 (Prša et al. 2016), allowing for a very homogenous analysis of the full sample.

Ideally, uniform space-based high-precision mediumcadence photometry and homogenous high-resolution highstability spectroscopic ground-based data for all the sample would suit the purpose of the very precise SBC calibration best. Such light curves will become available for many of the systems here if the TESS mission is successfully launched. We see this as a distant-future next natural step that will result in additional improvements of the internal consistency and precision of the derived physical parameters.

\subsection{Sample Enlargement}

In order to increase the number of eclipsing binaries with suitable data for this program, we have selected a number of additional suitable detached eclipsing binary systems and have collected spectroscopic and photometric data for them. They cover a wide range of spectral classes from B to early-K type, and they are mostly within $300 \mathrm{pc}$ from the Sun (low-extinction regime). In addition to AL Ari, for which we have derived physical parameters and a paper describing the full analysis will be published soon (P. Konorski et al. 2017, in preparation), our ongoing analysis is in an advanced stage for about 20 more systems.

The sample will be expanded in the near future with systems that have more precise Gaia parallaxes within and beyond a $300 \mathrm{pc}$ horizon. More systems will also join the sample from efforts of other research groups investigating eclipsing binary stars, as a large number of high-quality light curves from ground-based surveys (e.g., Super-WASP, soon the LSST) and space-based surveys (e.g., Kepler-2, soon TESS) is registered for both known and newly discovered systems. These efforts will surely result in significantly enlarging the sample to about 100 systems that cover B-, A-, F-, and G-type stars. More systems will not only help reduce statistical errors of the relations, but also determine the intrinsic spread of the SBC relations.

\subsection{Parallaxes}

Future more precise Gaia parallaxes are fundamental for any significant improvement of the SBC relations presented here. We forecast the expected precision of Gaia parallaxes for the sample as follows. We assumed conservatively that the precision of astrometry for bright stars (3 mag $<G<12 \mathrm{mag}$ ) will be $15 \mu$ as and that the photocenter movement of an eclipsing binary will be unequivocally detected and taken into account when it is larger than $35 \mu$ as. The resulting expected mean relative precision of Gaia parallaxes will be $0.6 \%$ for our sample. Systematic uncertainty introduced into the prediction of angular diameters will likely be smaller, but to be conclusive on this point, we need to wait for a final Gaia release quality evaluation. Figure 7 presents the expected angular diameters of stars in our sample after the final Gaia data release, assuming the same radii and uncertainties as in Table 3. Inspection of this figure suggests that much improvement is expected, especially for blue stars. Angular diameters with a precision better than $1 \%$ will be available for more than half of all components in our eclipsing binary sample. We add that the sample will be augmented by very precise dynamical parallaxes from interferometric orbits for a number of long-period eclipsing binary stars.

\subsection{Disentangling of Component Magnitudes}

It is interesting to estimate to which extent our extrapolation procedure introduces a bias. As we described in Section 3.7, flux ratios are calculated using precomputed intensities based on ATLAS9 atmosphere models, which assume a planeparallel geometry and local thermodynamic equilibrium (LTE). When components have similar effective temperatures to within about $100 \mathrm{~K}$, their light ratio changes very little over the optical and NIR range of the spectrum, and regardless of the adopted model atmosphere, the extrapolation leads to negligible errors in comparison with observational photometric uncertainties. However, the situation is somewhat different when the temperature difference between the components is much larger, say, on the order of $1000 \mathrm{~K}$.

For A-, F-, and G-type stars with given atmospheric parameters $\left(T_{\text {eff }}, \log g,[\mathrm{Fe} / \mathrm{H}]\right)$ and solar-like compositions the absolute spectral energy distributions predicted by various atmospheric models (plane parallel, spherical, LTE, and nonLTE) in a range of $B$ and $K$ bands differ by up to $5 \%$, but this 

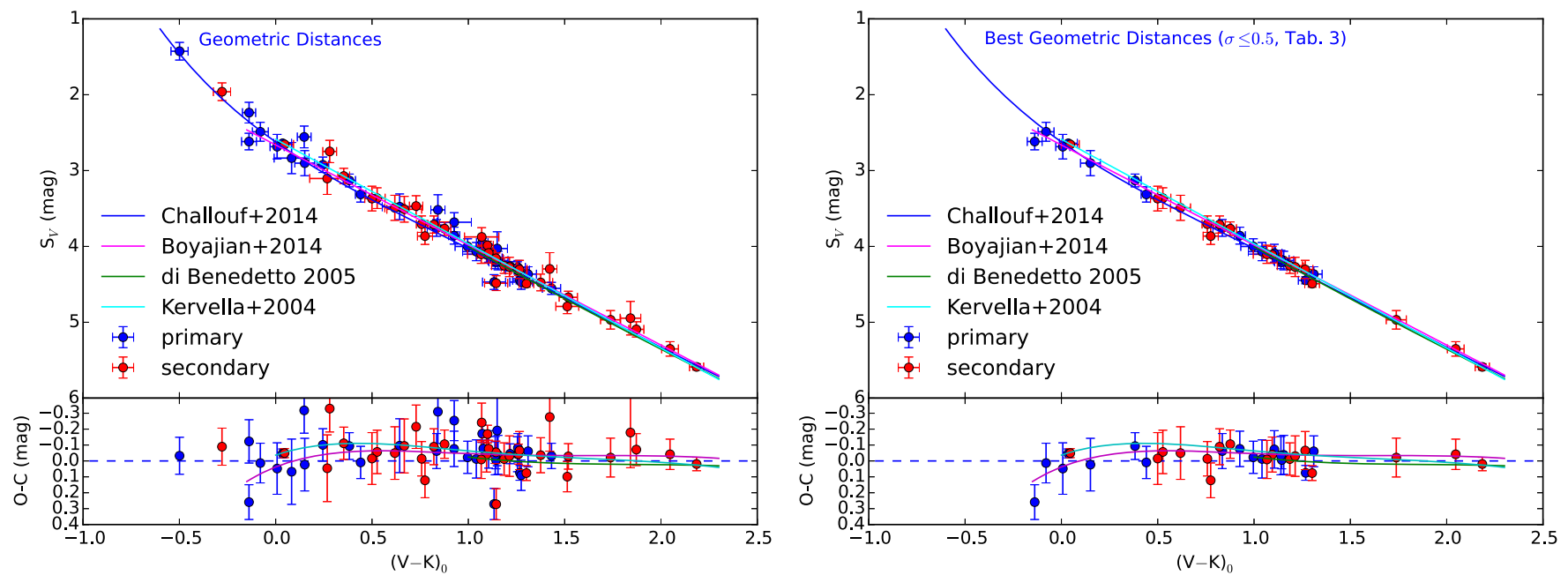

Figure 3. $V$-band surface brightness vs Johnson color $V-K$ relation. Left panel: for all stars based on their geometric distances. Right panel: for 14 systems with best agreement between the geometric and the photometric distances. Continuous lines correspond to several published interferometric SBC relations. The lower panels show O-C residuals calculated with respect to the SBC relation by Challouf et al. (2014), plotted as the dashed line.
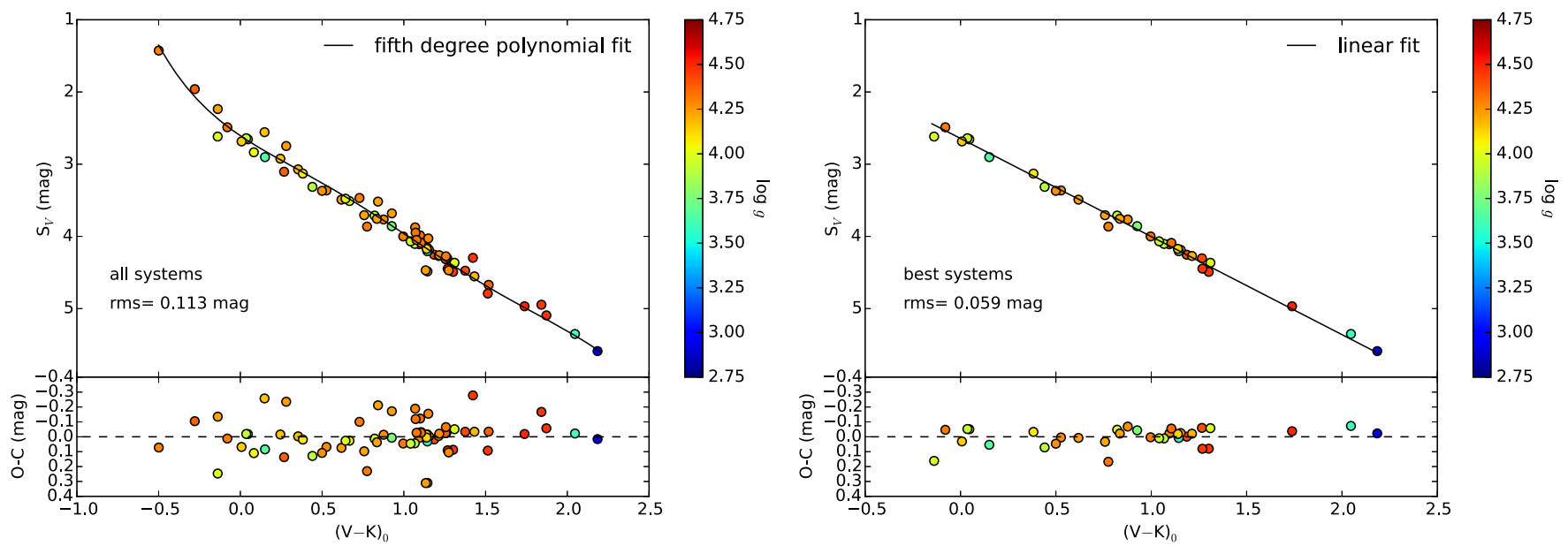

Figure 4. $V$-band surface brightness vs Johnson color $V-K$ relation with the addition of the surface gravity color scale (right vertical axis). The left panel shows all systems. The right panel shows the systems with the best-fit distances.

Table 5

Coefficients of Polynomial Fits to the Surface Brightness Parameter $S$ in $B$ and $V$ Bands

\begin{tabular}{lccccccccc}
\hline \hline Band & $\begin{array}{c}\text { Color } \\
\text { Index }\end{array}$ & $N^{\text {a }}$ & $\begin{array}{c}\text { Range of Color } \\
(\mathrm{mag})\end{array}$ & $a_{0}$ & $a_{1}$ & $a_{2}$ & $a_{3}$ & $a_{4}$ & $a_{5}$ \\
$(\%)$
\end{tabular}

Notes. The $S$ parameter is defined by Equation (3). Colors are in the Johnson photometric system. The limb-darkened stellar angular diameter is expressed in milliarcseconds and follows from the equation $\log \theta_{\mathrm{LD}}=0.2 *\left(a_{0}-m+a_{1} * X+\ldots+a_{5} * X^{5}\right)$, where $m$ is the observed extinction-free magnitude of a star in the $B$ or $V$ band, and $X$ is an extinction-free color. The last column gives the precision in predicting the angular diameter of stars in the given color range.

${ }^{a}$ Number of stars used in the fit.

difference is significantly smaller for the relative fluxes (i.e., colors) (e.g., Bessell et al. 1998; Martins \& Coelho 2007; Edvardsson 2008; Plez 2011). Comparison of model fluxes with empirical fluxes in the aforementioned range of the spectrum gives also very good agreements. As a result, we can expect on average a small systematic uncertainty in the derived 


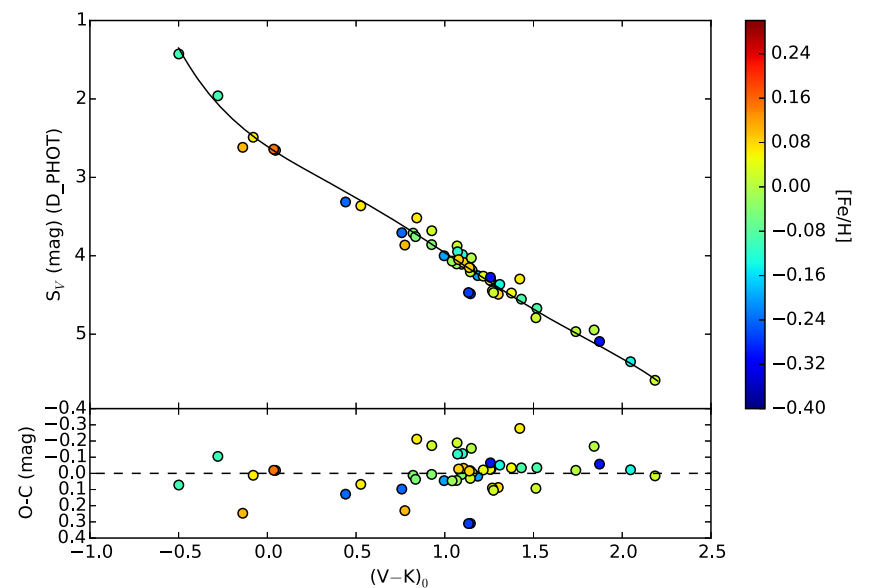

Figure 5. $V$-band surface brightness vs Johnson color $V-K$ for systems with determined metallicity. The continous line represents a fifth-order polynomial fit to the entire sample.

colors (reaching up to $0.02 \mathrm{mag}$ ) even in cases of larger temperature differences between the components. Such an error would be only a fraction of the typical uncertainty of an intrinsic color. This uncertainty can be mitigated even further by using multiband photometry and carefully determined temperatures derived from disentangled spectra. For hotter stars (O and B type), use of plane-parallel and LTE models may lead to much larger systematic shifts (e.g., Aufdenberg et al. 1998; Cugier 2012), but these issues will be addressed in a forthcoming paper.

\subsection{Photometry and Transformations into Standard System}

\subsubsection{Optical}

The precision of the transformation between the Tycho- 2 and Johnson photometric systems is about 1\% (Bessell 2000), resulting in additional systematic uncertainty in our SBC relations. To mitigate the problem, we would need to use original Tycho-2 $B_{T}$ and $V_{T}$ magnitudes and to express the calibration in this system. However, we note that for a few systems in our sample (EW Ori, VZ Hya, VZCep, LL Aqr, and EF Aqr), Tycho-2 photometry transformed into $B, V$ magnitudes gives optical and NIR colors that are inconsistent with each other and with the temperatures of the stars. In these cases we used other sources of $V$-band magnitudes. The source of discrepancy is unclear to us, but although the Tycho-2 photometry is multiepoch, in particular cases mean $B_{T}$ and $V_{T}$ magnitudes may be affected by eclipses and/or other types of systematics (e.g., transformation errors). This strengthens the case for well-calibrated, precise, and uniform optical $B, V$ photometry in the standard Johnson system for stars in the sample. In the optical, provided that a photometric system is close to the standard system, it is expected that transformation from an instrumental system to the standard system would not produce systematic errors larger than $0.5 \%$.

\subsection{2. $N I R$}

For the overwhelming majority of eclipsing binary systems, well-calibrated photometry NIR comes only from the singleepoch 2MASS survey. We transformed 2MASS magnitudes into the Johnson system, which may introduce systematics of up to $1 \%(0.02 \mathrm{mag})$ because of the poor definition of the
Johnson system in the NIR. As an example of this, the transformation equation for $(V-K)$ color used by Holmberg et al. (2007) has an offset of -0.02 mag with respect to the transformation equation we used, of course a non-negligible value for a precision better than $1 \%$. Preferentially, the future SBC calibration should be expressed in the 2MASS photometric system, which is well calibrated (e.g., Cohen et al. 2003) and is based on an all-sky network of standard stars, or possibly by using other NIR systems that have similar bandpasses and precisely determined transformation (e.g., SAAO).

Single-epoch photometry is prone to some accidental errors, and the statistical uncertainty of one measurement is relatively large. Because of this, it would be advisable to carry out new high-quality multiepoch NIR photometry for stars in the sample. This would significantly help to reduce statistical uncertainties and to remove any accidental photometric errors. We have started a campaign to secure NIR photometry for southern and equatorial stars from the sample with the plan to derive precise out-of-eclipse magnitudes and later also to provide full NIR light curves for some eclipsing binaries, especially those with a large effective temperature difference between components.

\subsection{Quantifying Error Contributions}

1. Radii: The mean precision of stellar radii determination in our sample is $1.2 \%$. If we were to use about 100 systems, then it would be possible to reduce the statistical error by a factor of 10 , i.e., to $0.1 \%-0.2 \%$. Systematics will come mostly from the numerical tools for the analysis of eclipsing binary stars, and it is expected to be on the order of $0.1 \%$.

2. Parallaxes: Taking into account the photocenter movements of the eclipsing binaries, the mean expected precision of Gaia parallaxes would be $0.6 \%$. The systematic error is expected to be significantly smaller.

3. Disentangling of magnitudes: Up to $0.01 \mathrm{mag}$ of the systematics in derived colors and magnitudes translates into a $0.3 \%$ mean systematic uncertainty in predicting angular diameters. However, if we were to use full NIR light curves and/or equal-temperature systems, then this error could be almost eliminated because it would be possible to determine the NIR magnitudes directly.

4. Photometric zero-points and transformations: In the best cases of well-defined photometric systems (Section 5.5), we expect $0.7 \%$ systematics in colors and magnitudes.

5. Interstellar extinction:

(a) Total extinction: the reddening is low for almost all our systems. When we assume a standard Galactic exctinction curve with $R_{V}=3.1$, it introduces only a little additional uncertainty of about $0.03 \mathrm{mag}$ in the $(V-K)_{0}$ color. Because the reddening line is largely parallel to the SBC relation, this translates into only a 0.006 mag statistical uncertainty $(0.3 \%)$ in predicting the angular diameter.

(b) Reddening law: for about $25 \%$ of stars in within $1 \mathrm{kpc}$ from the Sun (e.g., Fitzpatrick \& Massa 2007; Krełowski \& Strobel 2012), we expect deviations from the universal law. $R_{V}$ can vary significantly, but it mostly lies between 2.7 and 3.7 (e.g., Gontcharov 2012). When this is not accounted for, it shows as an additional intrinsic scatter in the SBC relation that amounts to about $0.02 \mathrm{mag}$ in some individual cases. 

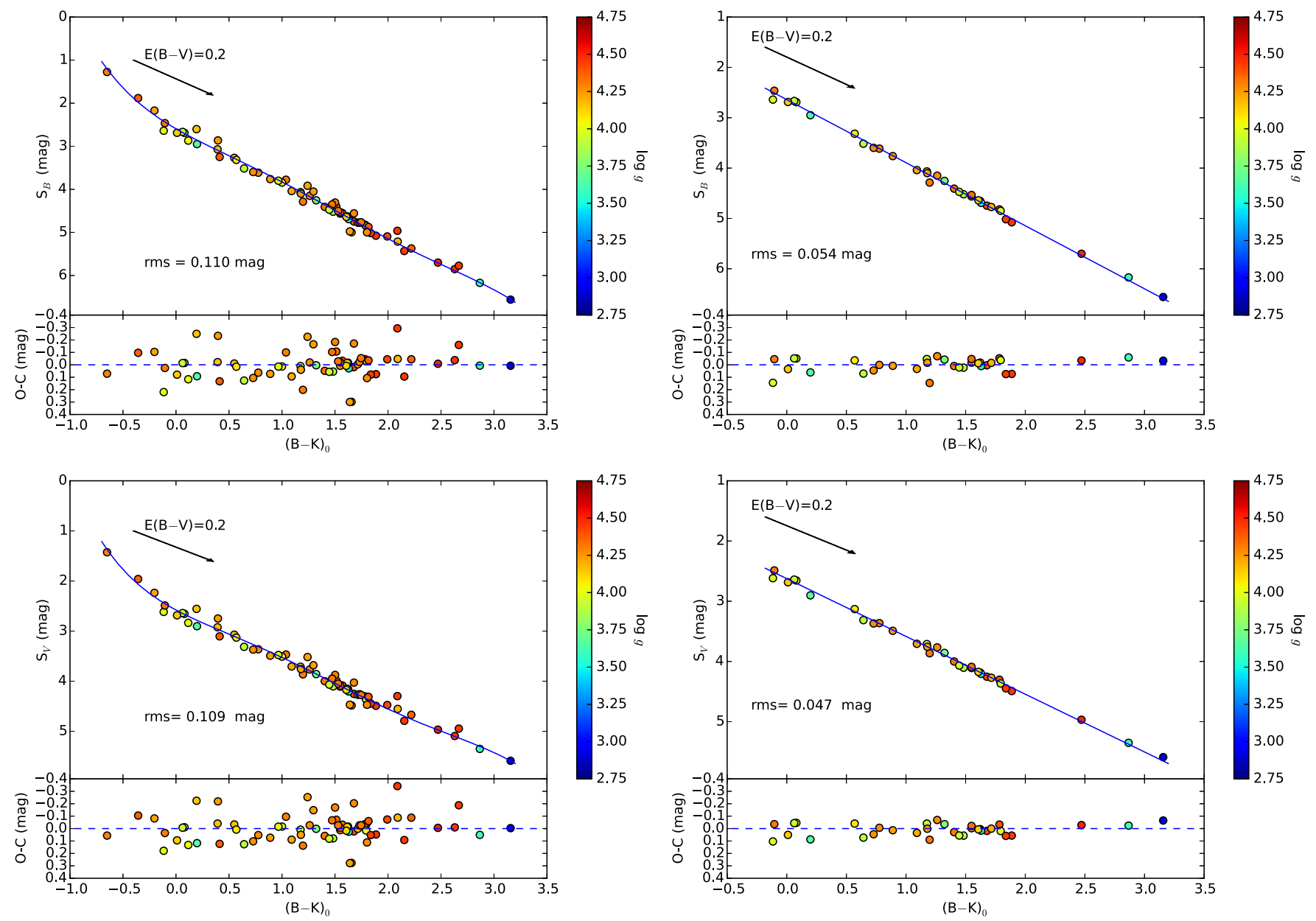

Figure 6. Surface brightness vs. Johnson color $B-K$ relations. Upper panel: calibrated for the $B$ band. Lower panel: calibrated for the $V$ band. The continuous line in the left panels shows fifth-order polynomial fits to all stars in the sample, and in the right panels the line shows linear fits to the best-fit sample. The reddening vector is denoted as an arrow. The root mean square of the relations is given. The surface gravity in cgs units is color coded.

The statistical uncertainty of the future SBC relation is expected to be well below $1 \%$ provided there are enough suitable systems (about 100 systems) and the internal dispersion of a given relation is low. By combining all conservative estimates of errors from the above considerations in quadrature, we obtain an upper limit of $0.9 \%$ on the systematic uncertainty. This error is dominated by the photometric uncertainties.

\section{Final Remarks}

New Gaia parallaxes combined with Hipparcos and dynamical parallaxes allow us to derive for the first time the SBC relations based fully on the eclipsing binary stars. The precision of the derived relations for A-, F-, and G-type stars is comparable to the precision of relations derived from interferometric angular diameters, and both types of relations are mutually consistent. The eclipsing binary method has no serious limitations if it is based on a well-selected sample of eclipsing binaries, a self-consistent analysis method, and proper sanity checks. To expand the SBC relation to O- and B-type stars, we propose to use the $B-K$ color, which allows reducing interstellar extinction uncertainties. We also discussed all the steps necessary to obtain precise and accurate SBC relations that allow for a precision better than $1 \%$ of the angular diameter predictions in the future.
The research leading to these results has received funding from the European Research Council (ERC) under the European Union's Horizon 2020 research and innovation program (grant agreement No 695099).

We are grateful for financial support from the Polish National Science Center grant MAESTRO 2012/06/A/ST9/ 00269. Support from the BASAL Centro de Astrofísica y Tecnologías Afines (CATA) PFB-06/2007, the Millenium Institute of Astrophysics (MAS) of the Iniciativa Cientifica Milenio del Ministerio de Economia, Fomento y Turismo de Chile, project IC120009, and the IdP II 2015000264 grant of the Polish Ministry of Science and Higher Education is also acknowledged. We also owe thanks to the staffs of the La Silla Observatory (ESO) and the Las Campanas Observatory (Carnegie) for their excellent support.

We also thank the anonymous referee for remarks and corrections to the text.

This research has made extensive use of the excellent astronomical SIMBAD database and of the VizieR catalog access tool, operated at CDS, Strasbourg, France, and we made also use of NASA's Astrophysics Data System Bibliographic Services (ADS).

This publication makes use of data products from the Two Micron All Sky Survey, which is a joint project of the University of Massachusetts and the Infrared Processing and 


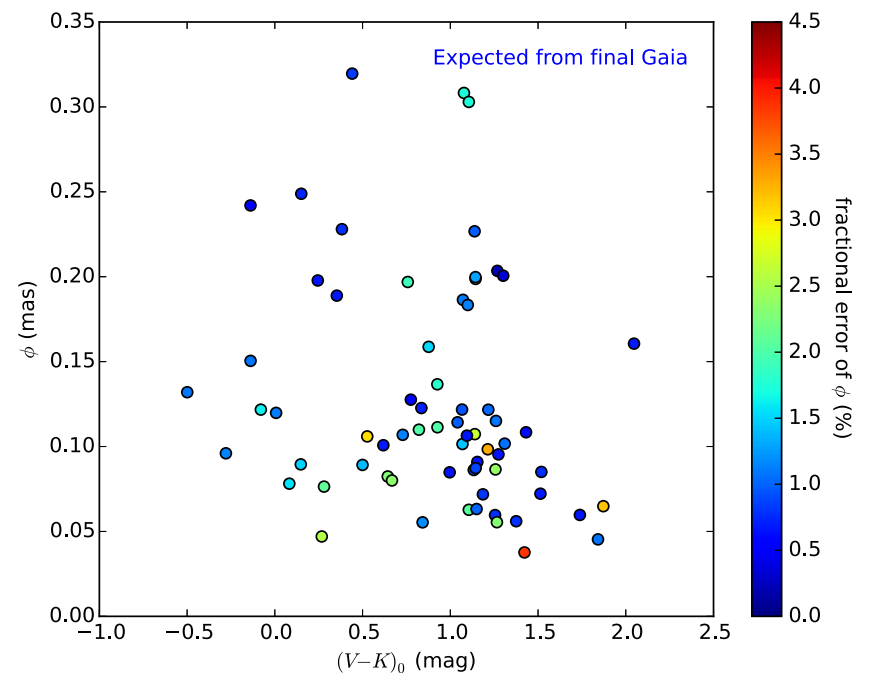

Figure 7. Predicted angular diameter uncertainties for stars in our sample after the final Gaia release. Note the change in scale of the color bar with respect to Figure 2. The error bars would in most cases be smaller than the size of the circles.

Analysis Center/California Institute of Technology, funded by the National Aeronautics and Space Administration and the National Science Foundation. We dedicate this work to professor Bohdan Paczyński who encouraged us many years ago to work on this subject.

\section{Appendix A \\ Temperatures and Reddening}

\section{A.1. V570 Per}

The temperature of the system V570 Per was determined from a model atmosphere analysis of disentangled spectra (Tomasella et al. 2008a). Although formal errors on the temperatures quoted by the authors are very small (lower than $0.5 \%$ ), the intrinsic colors of the components $b-y, B-V, V-J$, and $V-K$ point to much lower temperatures (by about $300 \mathrm{~K}$ ), unless there is significantly larger interstellar extinction to this object than assumed by Tomasella et al. (2008a): $E(B-V)=0.07 \mathrm{mag}$ instead of $0.023 \pm 0.007 \mathrm{mag}$. There are two ways of resolving the problem: (1) the temperatures are indeed lower, or (2) the reddening is indeed higher. The first possibility would force us to assume that some error was made by Tomasella et al. (2008a) during their atmospheric analysis. This seems quite unlikely, however: (a) their atmospheric analysis is standard, (b) the spectra are of good quality, (c) higher temperatures correspond well with the components' spectral types and masses. Thus the more probable explanation of disagreement is possibility (2). However, it was reported that the interstellar potasium line KI (7699 $\AA$ ) is not detected in the spectra of the system, which would contradict the higher reddening. Because we cannot solve this problem at the moment, for the purpose of this work, we kept the temperatures from Tomasella et al. (2008a) and assumed a reddening of $0.07 \pm 0.03 \mathrm{mag}$ to V570 Per. This problem clearly needs some future attention and more detailed investigation.

\section{A.2. WW Aur}

The temperatures of the components of WW Aur were previously determined by Smalley et al. (2002) and Southworth et al. (2005). However, $b-y, B-V$, and $V-K$ colors suggest temperatures higher by about $\sim 200 \mathrm{~K}$, which has been pointed out by Southworth et al. (2005) for the $b-y$ color. Wilson \& van Hamme (2009) used their direct distance estimate method and also found the temperatures of both components to be higher by a very similar amount. In our model we employed these higher temperatures.

\section{A.3. $\mathrm{KX} C n \mathrm{C}$}

For the system KX Cnc we determined the temperature from Strömgren uvby photometry $(b-y=0.378$; Olsen 1983) and Johnson's $B V J K$ photometry. The temperature of the primary component derived from the different colors is as follows: $T_{b-y}=5938 \mathrm{~K}, T_{B-V}=5985 \mathrm{~K}, T_{V-J}=6131 \mathrm{~K}$, and $T_{V-K}=$ $6162 \mathrm{~K}$. The resulting mean temperature is $T_{1}=6050 \mathrm{~K}$ i.e., higher by $150 \mathrm{~K}(1.5 \sigma)$ than the original temperature $T_{1}$ derived by Sowell et al. (2012). The higher value agrees better with the original HD spectral classification: F8 (Cannon \& Pickering 1918). Using the calibration between effective temperature and spectral type for normal main-sequence stars (Pecaut \& Mamajek 2013), we reclassify the system as F9V+F9V.

\section{A.4. RZ Cha}

The case of RZ Cha is interesting. Andersen et al. (1975) combined their velocimetry with Strömgren photometry obtained by Jørgensen \& Gyldenkerne (1975) to derive "mean" parameters of the components. The reason behind this was their conclusion that the components of the system had very similar physical appearance and thus also parameters. This "indistinguishability" of components was retained by Torres et al. (2010) in their review. However, it is clear from inspection of the light curves that the components have different surface temperatures, which has been reported by Giuricin et al. (1980). The difference is small, with the more massive and larger star being cooler by $\sim 50 \mathrm{~K}$, but it has an effect on the predicted infrared light ratios.

\section{A.5. WZ Oph}

The system was quite recently analyzed by Clausen et al. (2008a). They reported the temperature $T_{1}=6165 \pm 100 \mathrm{~K}$, based on reddening $E(B-V)=0.044 \mathrm{mag}$, intrinsic Strömgren color of the primary $(b-y)_{0}=0.329$, and a calibration by Holmberg et al. (2007). They noted that the temperature derived from atmospheric analysis of the disentangled primary's spectrum is slightly higher, but they did not report by how much. From unreddened colors $b-y, B-V, V-K$ we also derived a higher temperature of $T_{1}=6301 \mathrm{~K}(1.4 \sigma$ difference). Lower reddening of $E(B-V)=0.030$ mag resulting from Schlegel et al. (1998) maps leads to the temperature $T_{1}=6232 \mathrm{~K}$, a value that we would consider "slightly" higher. These reddening and temperature values are assumed in this work.

\section{A.6. UZ Dra}

Using $B, V, J, K$ photometry, we redetermined temperatures of the two components because the original temperatures given by Lacy et al. (1989) were estimated only from the mean spectral type of the system. The resulting temperatures are higher by about $200 \mathrm{~K}$ than those reported by Lacy et al. (1989) 
and correspond much better with the masses of the two components, which seem to be unevolved main-sequence stars.

\section{A.7. VZ Cep}

There is an inconsistency between the temperatures based on $B-V, b-y$ colors and $V-K, V-J$ with the NIR colors resulting in temperatures higher by about $300 \mathrm{~K}$. Different values of reddening do not resolve the discrepant temperatures. A possible reason is that $2 \mathrm{MASS}$ magnitudes are somehow affected, but they were taken well outside of eclipses and all have an "A" flag. Higher temperatures would be in agreement with relatively massive components of the system, and furthermore, the resulting photometric distance would be in perfect agreement with Hipparcos and Gaia parallaxes. However, we have no clue at this moment about the possible source of the discrepancy. We therefore retained in this paper the temperatures from Torres \& Lacy (2009), which are based on Strömgren photometry.

\section{Appendix B Radial Velocities}

\section{B.1. V570 Per}

Tomasella et al. (2008a) did not report radial velocity semiamplitudes. We used data from their Table 2 to rederive the orbital parameters. Our semimajor axis is larger by $1.5 \sigma$ than the value by Tomasella et al. (2008a), which we attribute mostly to a different choice of astrophysical constants, but our mass ratio $q$ is fully consistent with their value.

\section{B.2. HD 71636}

Henry et al. (2006) reported two sets of radial velocity semiamplitudes in their Tables 3 and 5 that contradict each other. We therefore rederived the spectroscopic orbit from the data in their Table 2. Our semiamplitudes are in perfect agreement with the values presented in Table 3 , and we accordingly adopted them here.

\section{B.3. $\mathrm{KX} \mathrm{Cnc}$}

Sowell et al. (2012) reported two sets of $K_{1,2}$ that contradict each other. Using the data from their Table 2, we determined a spectroscopic orbit that is fully consistent with the solution given in their Table 3.

\section{B.4. V4089 Sgr}

Recently, Veramendi \& González (2015) presented light and radial velocity curves solutions of the system, and they derived its absolute dimensions. However, the semimajor axis $a$ reported in their Table 1 is inconsistent with their radial velocity semiamplitudes $K_{1,2}$ and masses. Our solution to the velocimetry kindly provided by $\mathrm{M}$. Veramendi confirms their masses and $K_{1,2}$, but not their $a$. In addition, our $K_{2}$ is slightly larger (by $1.2 \sigma$ ); this is probably caused by the fact that we allowed for different systemic velocities for the components. Finally, we recalculated errors on the fundamental parameters that are significantly different from those reported in Tables 1 and 2 by Veramendi \& González (2015).

\section{B.5. EF Aqr}

Vos et al. (2012) presented fundamental physical parameters of the system. However, they reported two different sets of radial velocity semiamplitudes $K_{1,2}$ (their Tables 4 and 8). Using their velocimetry, we redetermined spectroscopic orbits for this system. Our $K_{1,2}$ are much closer to the values presented in Table 8, but they are still somewhat different. Especially the epoch of spectroscopic conjuction is different in our solution by 0.002 days, suggesting some period change in the system. We also recalculated fractional radii from the sum of radii and $k$ given in their Table 6 . The resulting radii and errors are again somewhat different from those reported in Table 6. Here we refer only to parameters that we have recalculated.

\section{B.6. V821 Cas}

Çakirli et al. (2009) reported their radial velocity measurements of the system. Their data in Table 1 are relatively noisy compared to present-day standards, nevertheless, we rederived the spectroscopic orbits in order to verify the consistency of the orbital parameters and quoted errors. The radial velocity semiamplitudes from our solution are marginally consistent with their values and the overall agreement of the orbit is satisfactory, also regarding the assumed errors.

\section{References}

Abt, H. A., \& Levato, H. 1978, PASP, 90, 201

Albrecht, S., Reffert, S., Snellen, I., Quirrenbach, A., \& Mitchell, D. S. 2007, A\&A, 474, 565

Alonso, A., Arribas, S., \& Martínez-Roger, C. 1996, A\&A, 313, 873

Ammons, S. M., Robinson, S. E., Strader, J., et al. 2006, ApJ, 638, 1004

Andersen, J. 1991, A\&Ar, 3, 91

Andersen, J., Clausen, C. V., Gustafsson, B., Nordström, B., \& VandenBerg, D. A. 1988, A\&A, 196, 128

Andersen, J., Clausen, J. V., \& Giménez, A. 1993, A\&A, 277, 439

Andersen, J., Clausen, J. V., \& Magain, P. 1989, A\&A, 211, 346

Andersen, J., Clausen, J. V., \& Nordström, B. 1987a, A\&A, 175, 60

Andersen, J., Clausen, J. V., Nordström, B., Tomkin, J., \& Mayor, M. 1991, A\&A, 246, 99

Andersen, J., García, J. M., Giménez, A., \& Nordström, B. 1987b, A\&A, 174,107

Andersen, J., Gjerløff, H., \& Imbert, M. 1975, A\&A, 44, 349

Andersen, J., \& Vaz, L. P. R. 1984, A\&A, 130, 102

Aufdenberg, J. P., Hauschildt, P. H., \& Baron, E. 1998, in ASP Conf. Ser. 131 (San Francisco, CA: ASP), 127

Bailer-Jones, C. A. L. 2015, PASP, 127, 994

Bakiş, V., Bakiş, H., Demircan, O., \& Eker, Z. 2008, MNRAS, 384, 1657

Barnes, T. G., Evans, D. S., \& Moffett, T. J. 1978, MNRAS, 183, 285

Batten, A. H., Fletcher, J. M., \& Mann, P. J. 1978, Publ. Dom. Astrophys. Obs., 15, 121

Behr, B. B., Cenko, A. T., Hajian, A. R., et al. 2011, AJ, 142, 6

Bessell, M. 2000, PASP, 112, 961

Bessell, M. S., \& Brett, J. M. 1988, PASP, 100, 1134

Bessell, M. S., Castelli, F., \& Plez, B. 1998, A\&A, 333, 231

Bilir, S., Ak, T., Soydugan, E., et al. 2008, AN, 117, 393

Boggs, P. T., \& Rogers, J. E. 1990, Orthogonal Distance Regression, Internal Report 89-419 (Gaithersburg, MD: National Institute of Standards and Technology)

Bonneau, D., Clausse, J.-M., Delfosse, X., et al. 2006, A\&A, 456, 789

Boyajian, T. S., van Belle, G., \& von Braun, K. 2014, AJ, 147, 47

Budding, E., Butland, R., \& Blackford, M. 2015, MNRAS, 448, 3784

Cannon, A. J., \& Pickering, E. C. 1918-1924, The Henry Draper Catalogue (Cambridge, MA: Ann. Astron. Obs. Harvard College), 91

Carpenter, J. M. 2001, AJ, 121, 2851

Casagrande, L., I. Ramírez, I., Meléndez, I., Bessell, J., \& Asplund, M. 2010, A\&A, 512, 54

Casertano, S., Riess, A. G., Bucciarelli, B., \& Lattanzi, M. G. 2016, arXiv:1609.05175v1 
Challouf, M., Nardetto, N., Mourard, D., et al. 2014, A\&A, 570, 104 Clausen, J. V., Bruntt, H., Olsen, E. H., Helt, B. E., \& Claret, A. 2010, A\&A, 511,22

Clausen, J. V., \& Grønbech, B. 1976, A\&A, 48, 49

Clausen, J. V., Torres, G., Bruntt, H., et al. 2008b, A\&A, 487, 1095

Clausen, J. V., Vaz, L. P. R., García, J. M., et al. 2008a, A\&A, 487, 1081

Cohen, M., Wheaton, Wm. A., \& Megeath, S. T. 2003, AJ, 126, 1090

Cugier, H. 2012, A\&A, 547, 42

David, T. J., Conroy, K. E., Hillenbrand, L. A., et al. 2016, AJ, 151, 112

de Bruijne, J. H. J., Rygl, K. L. J., \& Antoja, T. 2014, EAS Publ. Ser., 67, 23

Di Benedetto, G. P. 2005, MNRAS, 357, 174

Ducati, J. R. 2002, VizieR Online Data Catalog, 237, 0

Edvardsson, B. 2008, PhST, 133, 4011

Eker, Z., Bilir, S., Soydugan, F., et al. 2014, PASA, 31, 24

Fitzpatrick, E. L., \& Massa, D. 2007, ApJ, 663, 320

Flower, P. J. 1996, ApJ, 469, 355

Fouqué, P., \& Gieren, W. 1997, A\&A, 320, 799

Gaia Collaboration, Brown, A. G. A., Vallenari, A., Prusti, T., et al. 2016, A\&A, 595, 2

Gallenne, A., Pietrzyński, G., Graczyk, D., et al. 2016, A\&A, 586, 35

Gieren, W., Storm, J., Barnes, T. G., et al. 1995, ApJ, 627, 224

Giménez, A., \& Margrave, T. E. 1985, AJ, 90, 358

Giuricin, G., Mardirossian, F., Mezzetti, M., \& Predolin, F. 1980, A\&A, 85,259

Gontcharov, G. A. 2012, AstL, 38, 12

González Hernández, J. I., \& Bonifacio, P. 2009, A\&A, 497, 497

Graczyk, D., Maxted, P. F. L., Pietrzyński, G., et al. 2015, A\&A, 581, 106

Graczyk, D., Pietrzyński, G., Thompson, I. B., et al. 2014, ApJ, 780, 59

Graczyk, D., Smolec, R., Pavlovski, K., et al. 2016, A\&A, 594, 92

Griffin, R. F. 2013, Observatory, 133, 156

Groenewegen, M. A. T., Decin, L., Salaris, M., \& De Cat, P. 2007, A\&A, 463,579

Guillout, P., Klutsch, A., Frasca, A., et al. 2009, A\&A, 504, 829

Gülmen, O., Güdür, N., \& Sezer, C. 1986, IBVS, 2953, 1

Habbereiter, M., Schmutz, W., \& Kosovichev, A. G. 2008, ApJ, 675, 53

Hełminiak, K. G., Konacki, M., Ratajczak, M., \& Muterspaugh, M. W. 2009, MNRAS, 400, 969

Henry, G. W., Fekel, F. C., Sowell, J. R., \& Gearhart, J. S. 2006, AJ, 132 2489

Hill, G., Hilditch, R. W., Younger, F., \& Fisher, W. A. 1975, MmRAS, 79, 131

Hindsley, R. B., \& Bell, R. A. 1989, ApJ, 341, 1004

Høg, E., Fabricius, C., Makarov, V. V., et al. 2000, A\&A, 357, 367

Holmberg, J., Nordström, B., \& Andersen, J. 2007, A\&A, 475, 519

Holmberg, J., Nordström, B., \& Andersen, J. 2009, A\&A, 501, 941

Houdashelt, M. L., Bell, R. A., \& Sweigart, A. V. 2000, AJ, 119, 1448

Houk, N. 1978, Michigan Catalogue of two-dimensional spectral types for HD stars, Vol. 2 (Ann Arbor, MI: Univ. of Michigan)

Houk, N., \& Cowley, A. P. 1975, Michigan Spectral Catalog, Vol. 1 (Ann Arbor, MI: Univ. of Michigan)

Houk, N., \& Swift, C. 1999, Michigan Catalogue of Two-dimensional Spectral Types for HD Stars, Vol. 5 (Ann Arbor: Univ. of Michigan)

Hrivnak, B. J., \& Milone, E. F. 1984, ApJ, 282, 748

Hummel, C. A., Armstrong, J. T., Buscher, D. F., et al. 1995, AJ, 110, 376

Çakirli, Ö., Ibanoğlu, C., Bilir, S., \& Sipahi, E. 2009, MNRAS, 395, 1649

Imbert, M. 1986, A\&AS, 65, 97

Imbert, M. 2002, A\&A, 387, 850

Jerzykiewicz, M. 2001, AcA, 51, 151

Jørgensen, H. E., \& Gyldenkerne, K. 1975, A\&A, 44, 343

Kervella, P., Thévenin, F., Di Folco, E., \& Ségransan, D. 2004, A\&A, 426, 297

Khaliullin, Kh. F., \& Khaliullina, A. I. 2002, ARep, 46, 119

Khaliullin, Kh. F., Khaliullina, A. I., \& Krylov, A. V. 2001, ARep, 45, 888

Kirkby-Knet, J. A., Maxted, P. F. L., Serenelli, A. M., et al. 2016, A\&A, 591,124

Kiyokawa, M., \& Kitamura, M. 1975, Ann. Tokyo Astron. Obs., 15, 117

Krełowski, J., \& Strobel, A. 2012, AN, 333, 60

Kruszewski, A., \& Semeniuk, I. 1999, AcA, 49, 561

Kurucz, R. 1993, ATLAS9 Stellar Atmosphere Programs and $2 \mathrm{~km} \mathrm{~s}^{-1}$ Grid (Cambridge, MA: SAO) Kurucz CD-ROM No. 13

Lacy, C. H., Gülmen, O., Güdür, N., \& Sezer, C. 1989, AJ, 97, 822

Lacy, C. H. S. 1977, ApJ, 213, 458

Lacy, C. H. S. 1981, ApJ, 251, 591

Lacy, C. H. S. 2002, AJ, 124, 1162

Lacy, C. H. S., Fekel, F. C., \& Claret, A. 2012, AJ, 144, 63

Lutz, T. E., \& Kelker, D. H. 1973, PASP, 85, 573
Lyubimkov, L. S., Rachkovskaya, T. M., \& Rostopchin, S. I. 1996, ARep, 40, 802

Malkov, O. Y. 1993, BICDS, 42, 27

Martins, L. P., \& Coelho, P. 2007, MNRAS, 381, 1329

Masana, E., Jordi, C., \& Ribas, I. 2006, A\&A, 450, 735

Maxted, P. F. L. 2016, A\&A, 591, 111

Maxted, P. F. L., Hutcheon, R. J., Torres, G., et al. 2015, A\&A, 578, 25

Mermilliod, J. C. 1991, Catalogue of Homogeneous Means in the UBV System (Geneva: Institut d'Astronomie, Universite de Lausanne)

Munari, U., Dallaporta, S., Siviero, A., et al. 2004, A\&A, 418, L31

Napiwotzki, R., Schoenberner, D., \& Wenske, V. 1993, A\&A, 268, 653

Nesterov, V. V., Kuzmin, A. V., Ashimbaeva, N. T., et al. 1995, A\&As, 110,367

Nordström, B., \& Johansen, K. T. 1994b, A\&A, 291, 777

Ochsenbein, F., Bauer, P., \& Marcout, J. 2000, A\&AS, 143, 23

Olsen, E. H. 1983, A\&As, 54, 55

Pavlovski, K., Southworth, J., Kolbas, V., \& Smalley, B. 2014, MNRAS, 438, 590

Pecaut, M. J., \& Mamajek, E. E. 2013, ApJS, 208, 9

Pietrzyński, G., Graczyk, D., Gieren, W., et al. 2013, Natur, 495, 76

Pitjeva, E. V., \& Standish, E. M. 2009, CeMDA, 103, 356

Plez, B. 2011, JPhCD, 328, 2005

Popper, D. M. 1965, ApJ, 141, 126

Popper, D. M. 1966, AJ, 71, 175

Popper, D. M. 1971, ApJ, 169, 549

Popper, D. M. 1980, ARA\&A, 18, 115

Popper, D. M. 1984, AJ, 89, 132

Popper, D. M. 1998, PASP, 110, 919

Popper, D. M., \& Etzel, P. B. 1981, AJ, 86, 102

Popper, D. M., Lacy, C. H., Frueh, M. L., \& Turner, A. E. 1986, AJ, 91, 383

Prša, A., Conroy, K. E., Horvat, M., et al. 2016, ApJS, 227, 29

Ramírez, I., \& Meléndez, J. 2005, AJ, 626, 465

Ratajczak, M., Kwiatkowski, T., Schwarzenberg-Czerny, A., et al. 2010, MNRAS, 402, 2424

Ribas, I., Jordi, C., \& Jordi, T. 1999, MNRAS, 309, 199

Sandage, A., \& Saha, A. 2002, AJ, 123, 2047

Schlegel, D. J., Finkbeiner, D. P., \& Davis, M. 1998, ApJ, 500, 525

Semeniuk, I. 2001, AcA, 51, 75

Skrutskie, M. F., Cutri, R. M., Stiening, R., et al. 2006, AJ, 131, 1163

Smalley, B., Gardiner, R. B., Kupka, F., \& Bessell, M. F. 2002, A\&A, 395, 601

Smith, B. 1948, ApJ, 108, 504

Southworth, J. 2013, A\&A, 557, 119

Southworth, J. 2015, in ASP Conf. Ser. 496 Living Together: Planets, Host Stars and Binaries, ed. S. Rucinski, G. Torres, \& M. Zejda (San Francisco, CA: ASP), 164

Southworth, J., Bruntt, H., \& Buzasi, D. L. 2007, A\&A, 467, 1215

Southworth, J., Smalley, B., Maxted, P. F. L., Claret, A., \& Etzel, P. B. 2005, MNRAS, 363, 529

Sowell, J. R., Henry, G. W., \& Fekel, F. C. 2012, AJ, 143, 5

Stassun, K. G., \& Torres, G. 2016, AJ, 152, 180

Stassun, K. G., \& Torres, G. 2016, ApJ, 831L, 6

Storm, J., Gieren, W., Fouqué, P., et al. 2011, A\&A, 534, 94

Suchomska, K., Graczyk, D., Smolec, R., et al. 2015, MNRAS, 451, 651

Tomasella, L., Munari, U., Cassisi, S., et al. 2008a, A\&A, 483, 263

Tomasella, L., Munari, U., Siviero, A, et al. 2008b, A\&A, 480, 465

Tomkin, J., \& Fekel, F. C. 2006, AJ, 131, 2652

Torres, G., Andersen, J., \& Giménez, A. 2010, A\&ARv, 18, 67

Torres, G., Andersen, J., Nordström, B., \& Latham, D. W. 2000, AJ, 119, 1942

Torres, G., \& Lacy, C. H. S. 2009, AJ, 137, 507

Tucker, R. S., Sowell, J. R., Williamon, R. M., \& Coughlin, J. L. 2009, AJ, 137, 2949

van Hamme, W., \& Wilson, R. E. 2007, ApJ, 661, 1129

van Leeuwen, F. 2007, A\&A, 474, 653

Veramendi, M. E., \& González, J. F. 2015, NewA, 34, 266

Vos, J., Clausen, J. V., Jørgensen, U. G., et al. 2012, A\&A, 540, 64

Wenger, M, Ochsenbein, F., Egret, D., et al. 2000, A\&As, 143, 9

Wilson, R. E. 1979, ApJ, 234, 1054

Wilson, R. E. 1990, ApJ, 356, 613

Wilson, R. E., \& Devinney, E. J. 1971, ApJ, 166, 605

Wilson, R. E., \& van Hamme, W. 2009, ApJ, 699, 118

Wilson, R. E., van Hamme, W., \& Terrell, D. 2010, ApJ, 723, 1469

Wolf, M., \& Zejda, M. 2005, A\&A, 437, 545

Worthey, G., \& Lee, H. 2011, ApJS, 193, 1

Wyithe, J. S. B., \& Wilson, R. E. 2002, ApJ, 571, 293 